\title{
Diboron-Carbene Complexes Derived from Geminal Dianion
}

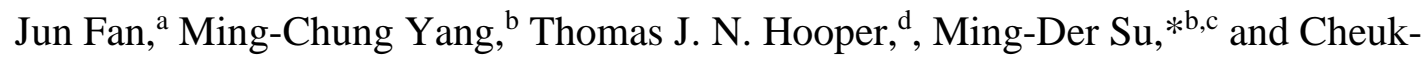
Wai So*a

${ }^{a}$ Division of Chemistry and Biological Chemistry, School of Physical and Mathematical Sciences, Nanyang Technological University, Singapore 637371. ' Department of Applied Chemistry, National Chiayi University, Chiayi 60004, Taiwan. 'Department of Medicinal and Applied Chemistry, Kaohsiung Medical University, Kaohsiung 80708, Taiwan. ${ }^{\mathrm{d} H i g h}$ Field NMR Facility, Division of Physics and Applied Physics, School of Physical and Mathematical Sciences, Nanyang Technological University, Singapore 637371

\section{Supporting Information}

Table of Contents

S1. Selected NMR Spectra and HRMS

S2. X-ray Data Collection and Structural Refinement

S3. Theoretical Studies

S4. References 


\section{S1. Selected NMR Spectra and HRMS}

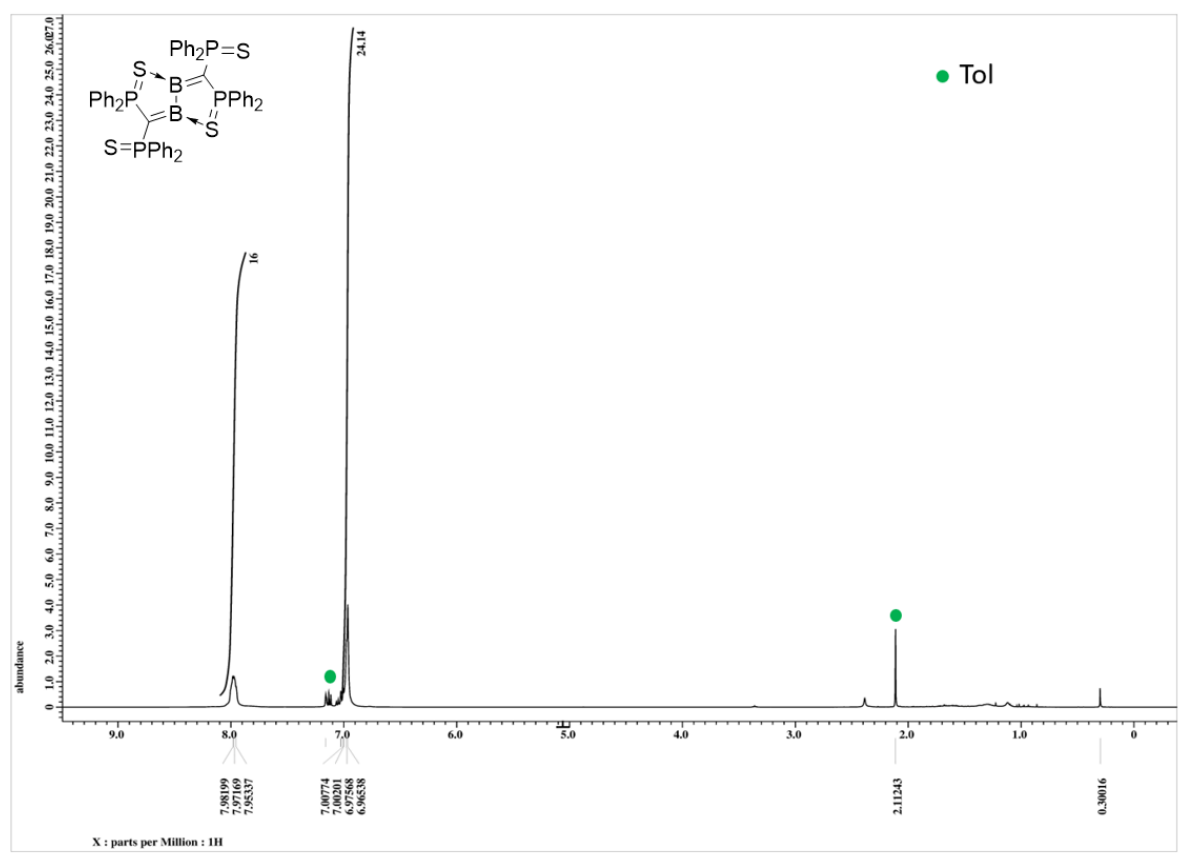

Figure S1. ${ }^{1} \mathrm{H}$ NMR spectrum of compound 2 (in $\mathrm{C}_{6} \mathrm{D}_{6}$ ). Signals at 0.3 and $0.9-1.3 \mathrm{ppm}$ correspond to silicone and $\mathrm{H}$ grease that were used to handle air- and moisture-sensitive compounds.

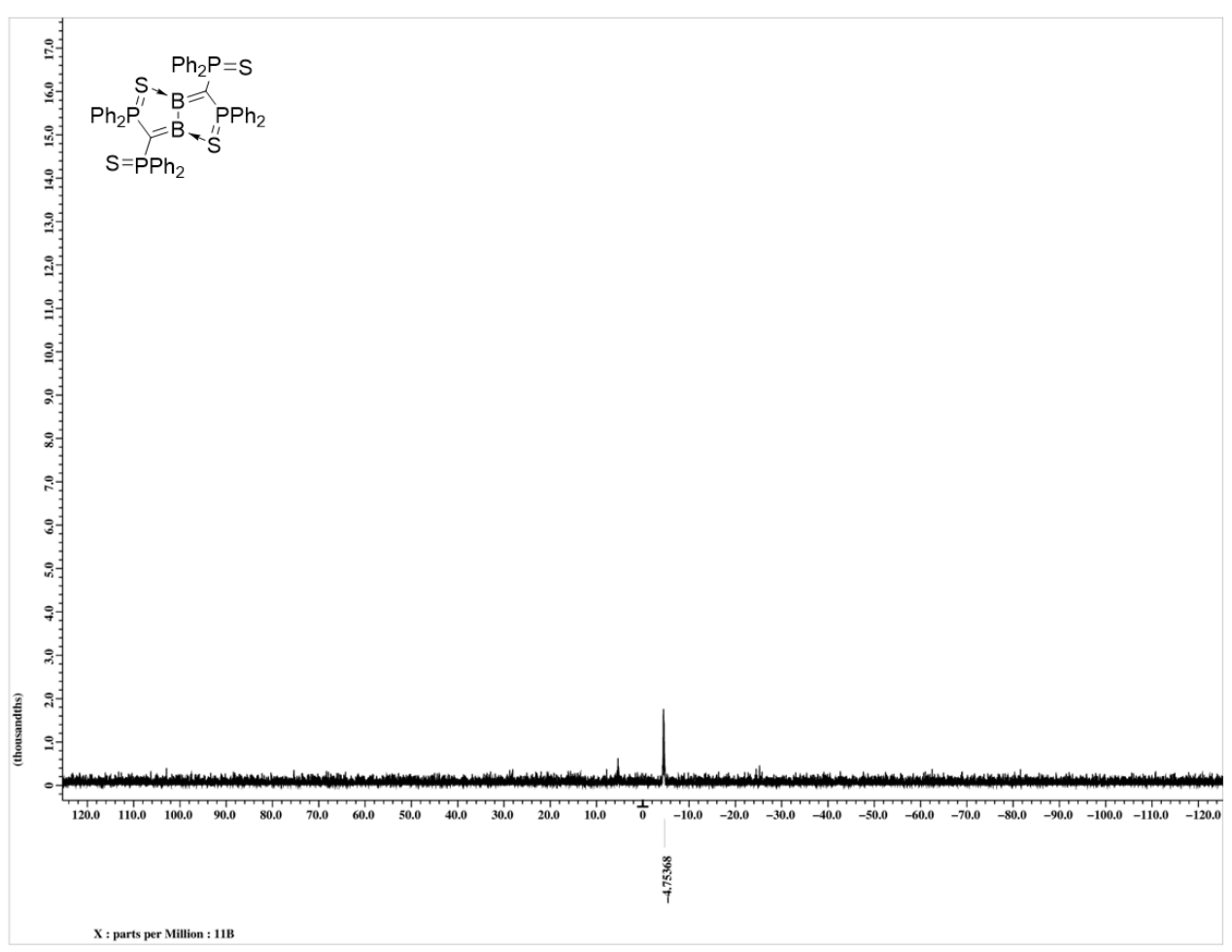

Figure S2. ${ }^{11} \mathrm{~B}\left\{{ }^{1} \mathrm{H}\right\}$ NMR spectrum of compound 2 (in $\mathrm{C}_{6} \mathrm{D}_{6}$ ). 


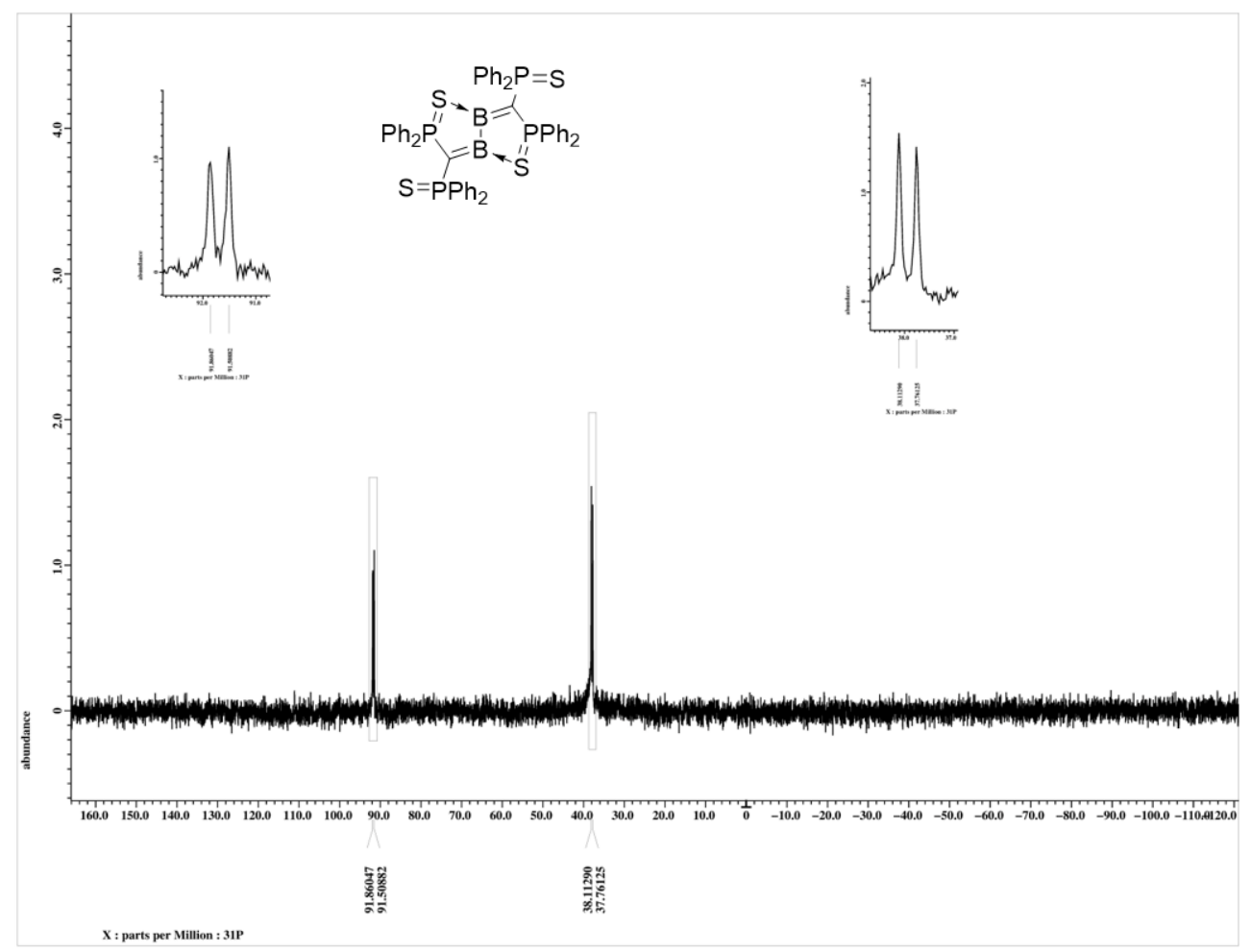

Figure S3. ${ }^{31} \mathrm{P}\left\{{ }^{1} \mathrm{H}\right\}$ NMR spectrum of compound 2 (in $\mathrm{C}_{6} \mathrm{D}_{6}$ ).

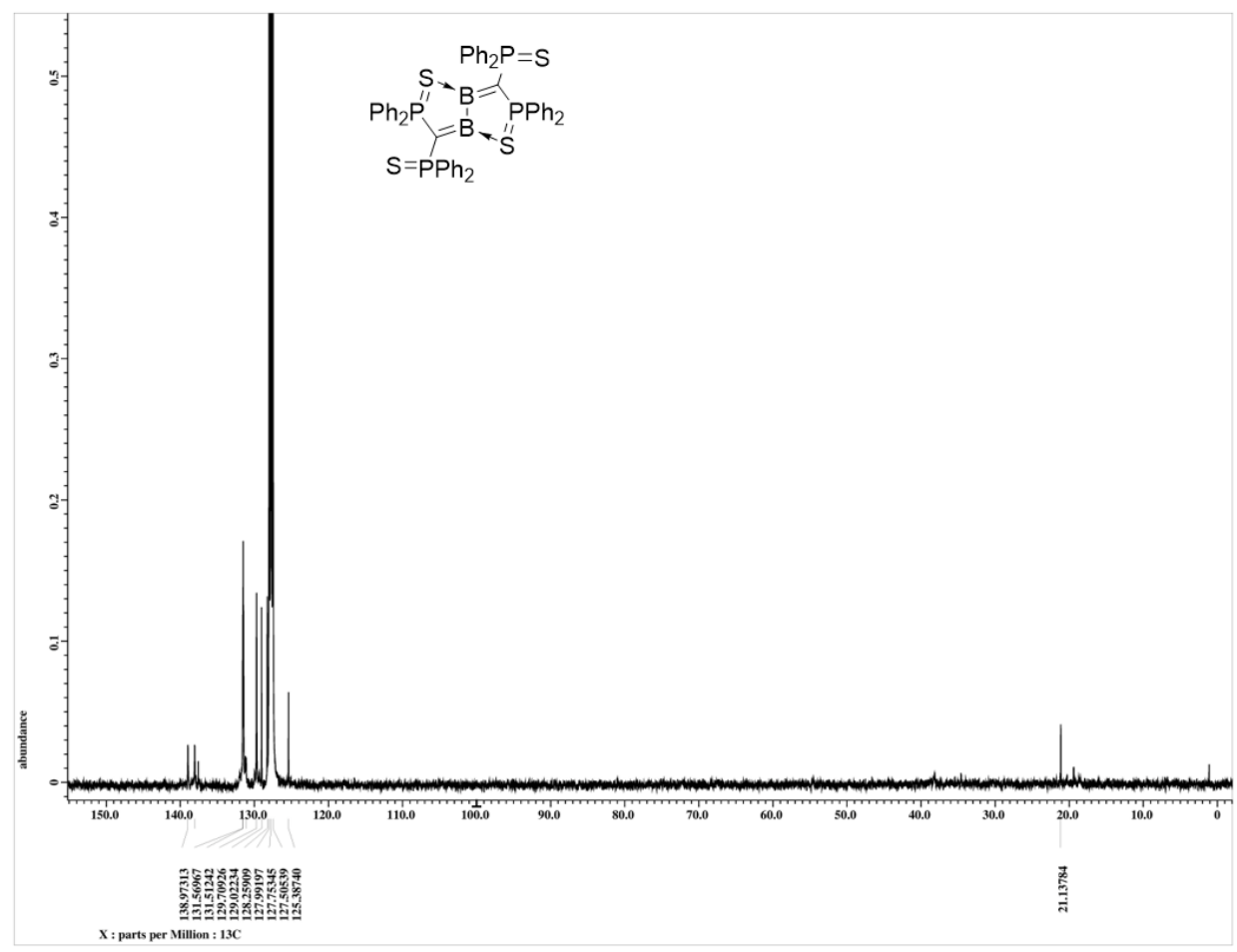

Figure S4. ${ }^{13} \mathrm{C}\left\{{ }^{1} \mathrm{H}\right\}$ NMR spectrum of compound 2 (in $\mathrm{C}_{6} \mathrm{D}_{6}$ ). 


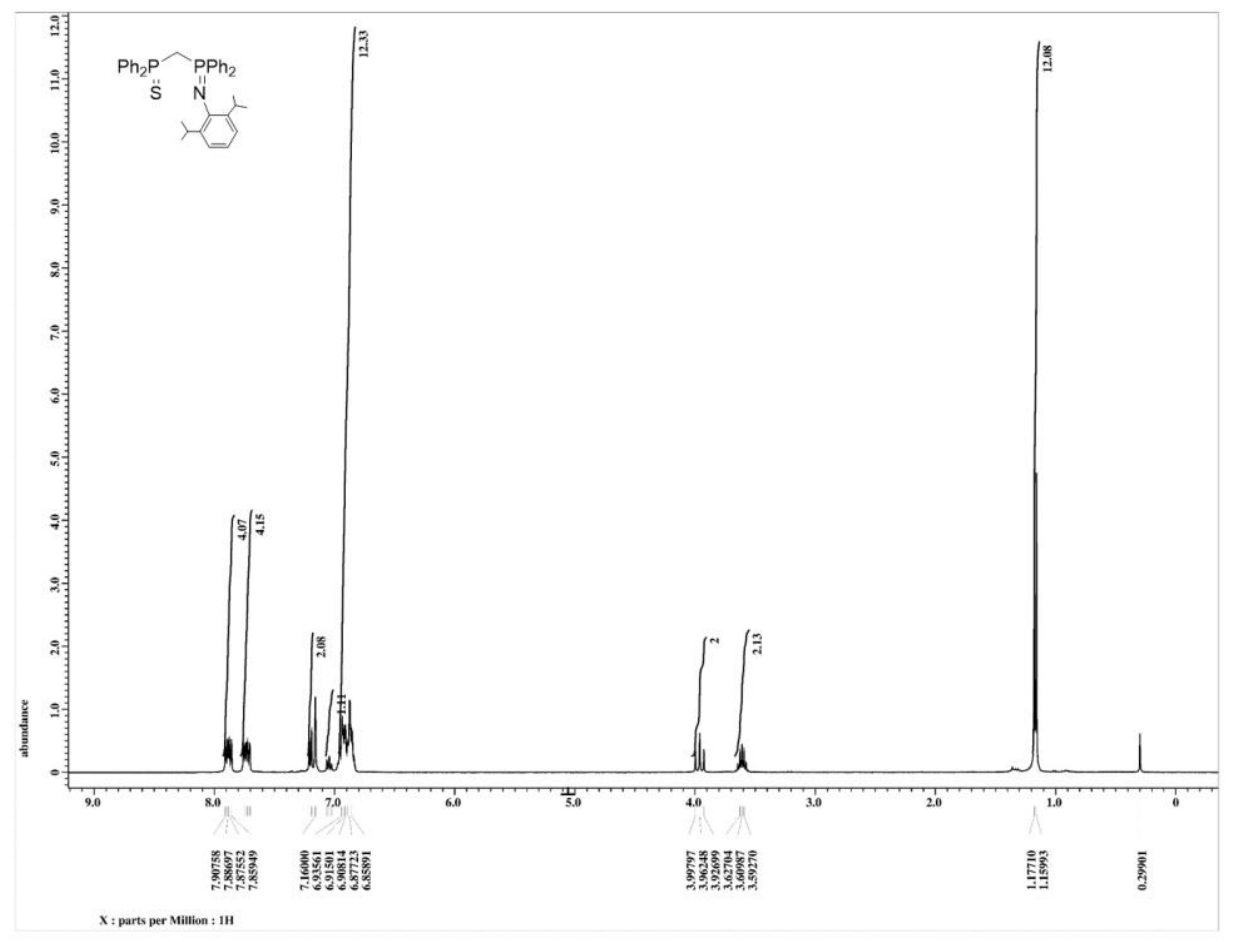

Figure S5. ${ }^{1} \mathrm{H}$ NMR spectrum of $\mathrm{H}_{2} \mathrm{C}\left(\mathrm{PPh}_{2} \mathrm{NAr}\right)\left(\mathrm{PPh}_{2} \mathrm{~S}\right)$ (in $\left.\mathrm{C}_{6} \mathrm{D}_{6}\right)$. Signals at 0.3 and $0.9-$ $1.3 \mathrm{ppm}$ correspond to silicone and $\mathrm{H}$ grease that were used to handle air- and moisturesensitive compounds.

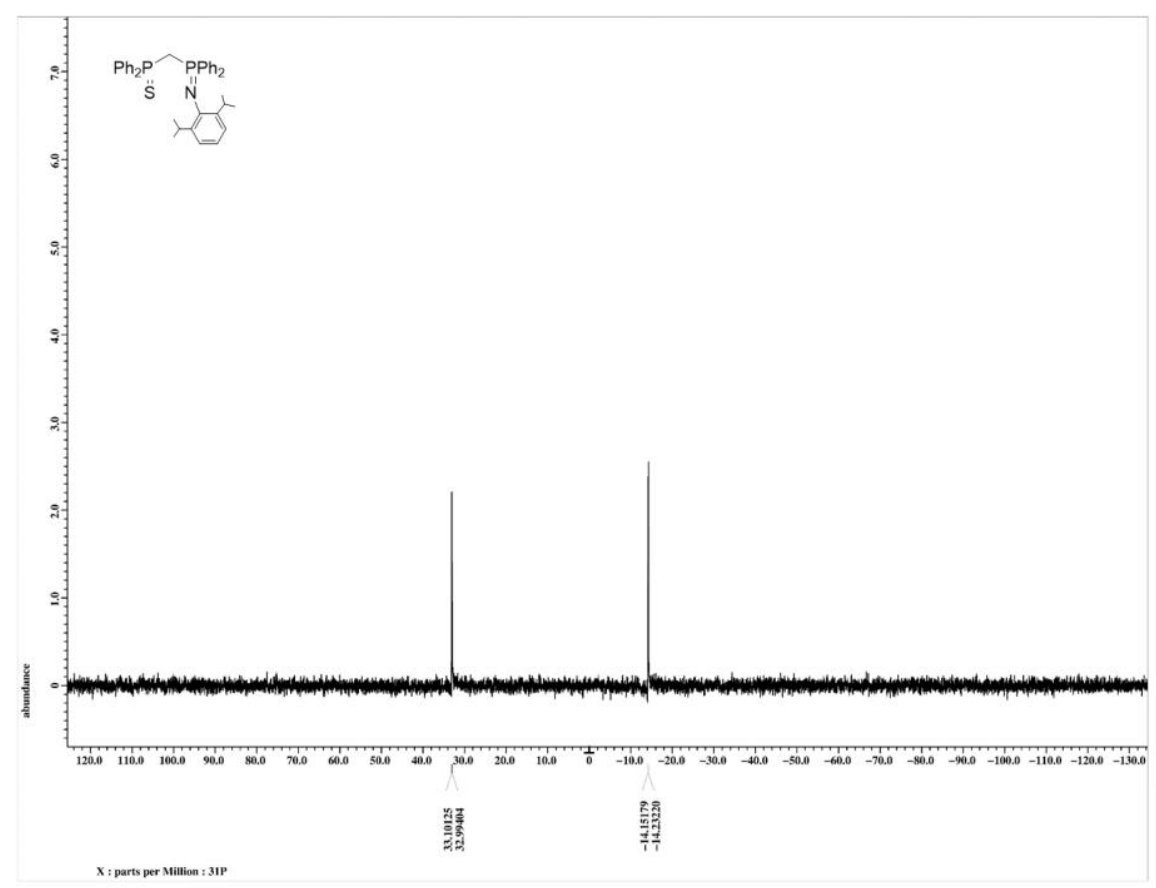

Figure S6. ${ }^{31} \mathrm{P}\left\{{ }^{1} \mathrm{H}\right\}$ NMR spectrum of $\mathrm{H}_{2} \mathrm{C}\left(\mathrm{PPh}_{2} \mathrm{NAr}\right)\left(\mathrm{PPh}_{2} \mathrm{~S}\right)$ (in $\left.\mathrm{C}_{6} \mathrm{D}_{6}\right)$. 


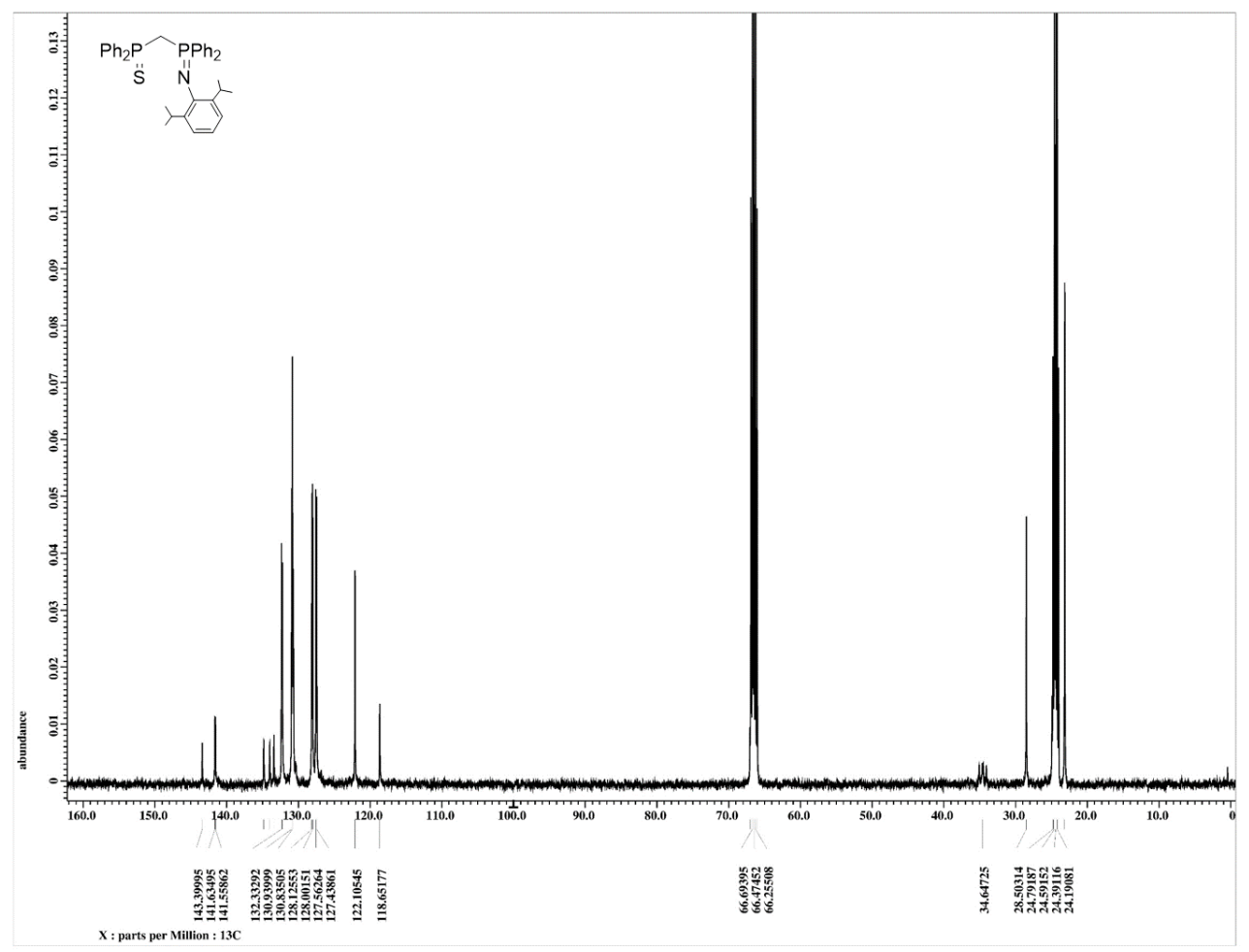

Figure S7. ${ }^{13} \mathrm{C}\left\{{ }^{1} \mathrm{H}\right\}$ NMR spectrum of $\mathrm{H}_{2} \mathrm{C}\left(\mathrm{PPh}_{2} \mathrm{NAr}\right)\left(\mathrm{PPh}_{2} \mathrm{~S}\right)$ (in $\left.\mathrm{THF}-\mathrm{d}_{8}\right)$.

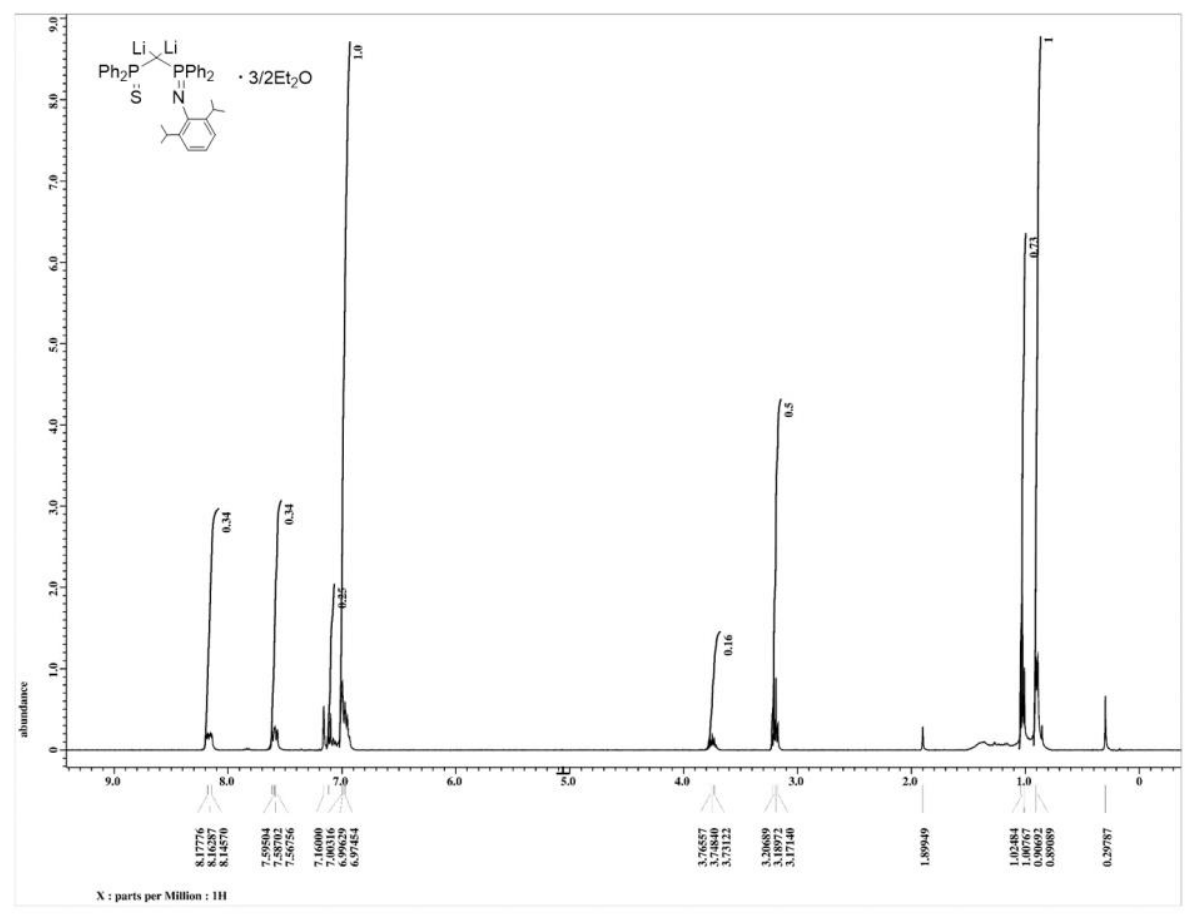

Figure S8. ${ }^{1} \mathrm{H}$ NMR spectrum of compound 3 (in $\mathrm{C}_{6} \mathrm{D}_{6}$ ). Signals at 0.3 and $0.9-1.3 \mathrm{ppm}$ correspond to silicone and $\mathrm{H}$ grease that were used to handle air- and moisture-sensitive compounds. 


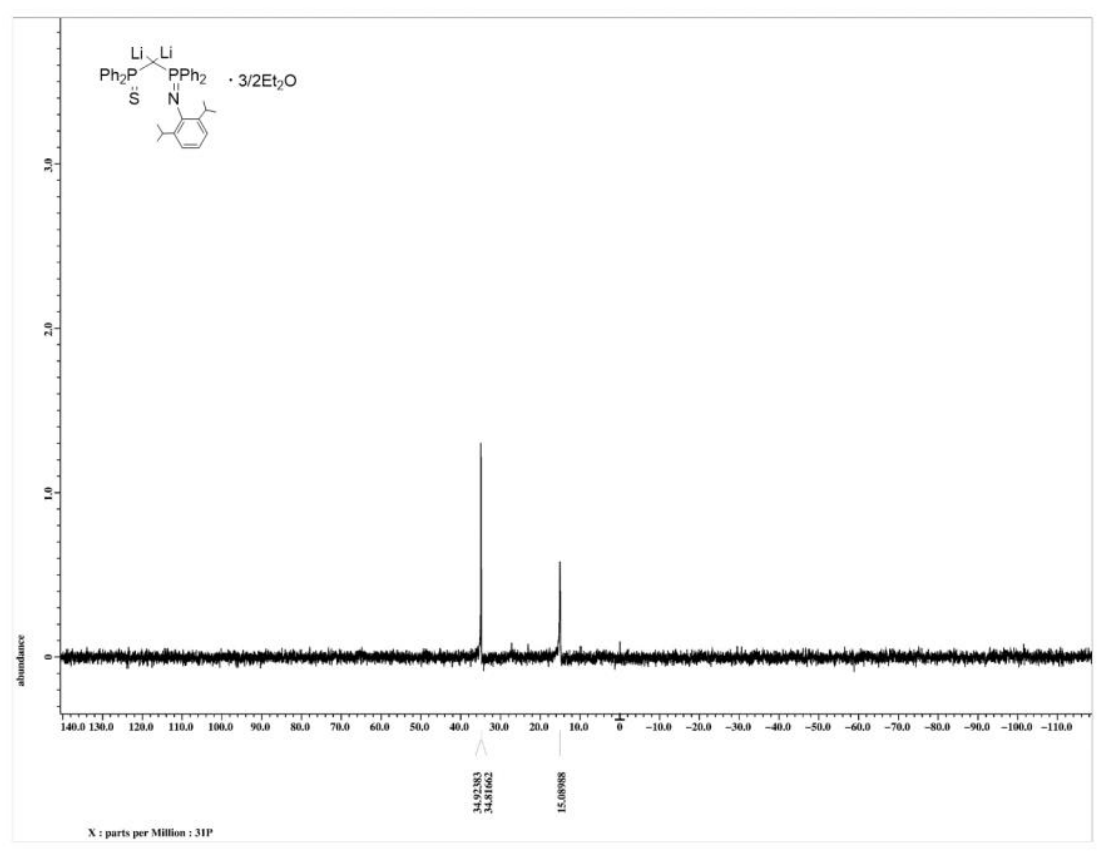

Figure S9. ${ }^{31} \mathrm{P}\left\{{ }^{1} \mathrm{H}\right\}$ NMR spectrum of compound 3 (in $\mathrm{C}_{6} \mathrm{D}_{6}$ ).

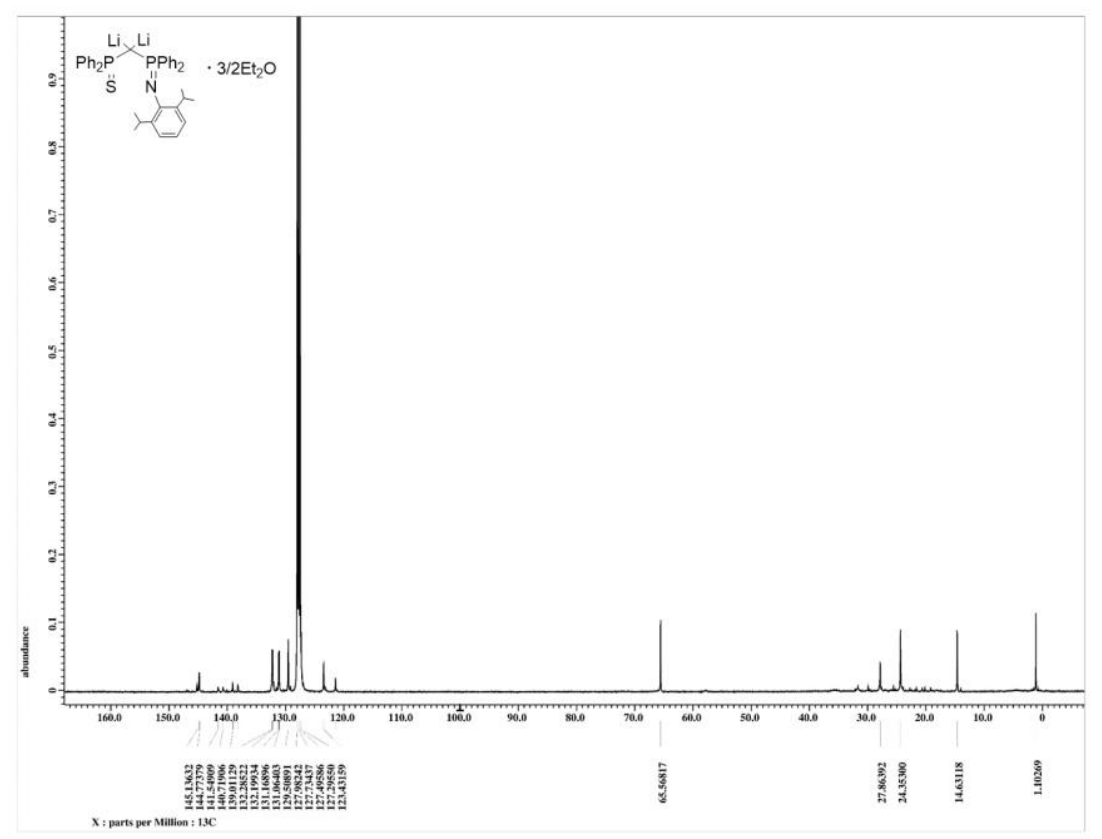

Figure S10. ${ }^{13} \mathrm{C}\left\{{ }^{1} \mathrm{H}\right\}$ NMR spectrum of compound $\mathbf{3}$ (in $\mathrm{C}_{6} \mathrm{D}_{6}$ ). 


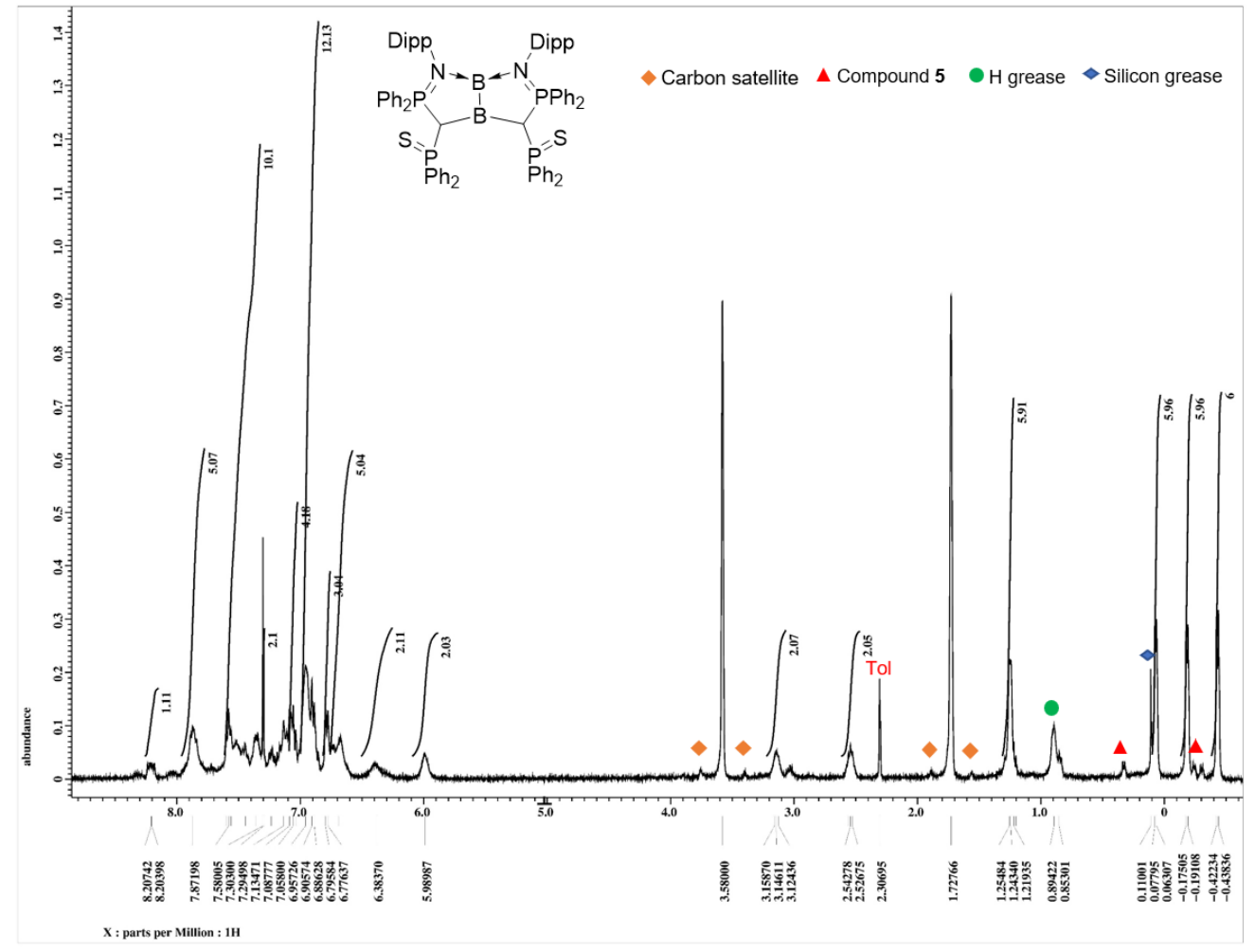

Figure S11. ${ }^{1} \mathrm{H}$ NMR spectrum of compound 4 (in THF- $\mathrm{d}_{8}$ ). Signals at 0.3 and $0.9-1.3 \mathrm{ppm}$ correspond to silicone and $\mathrm{H}$ grease that were used to handle air- and moisture-sensitive compounds.

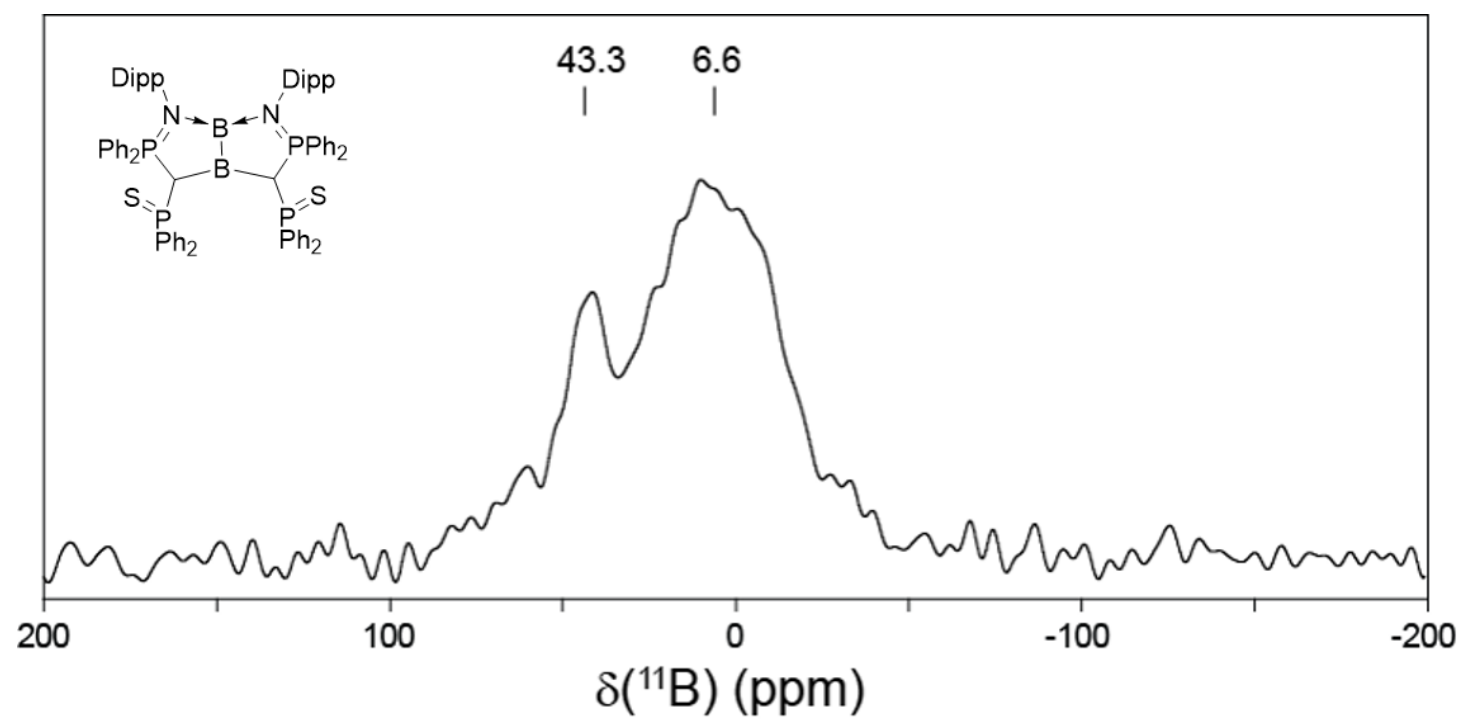

Figure S12. ${ }^{11}$ B MAS NMR spectrum of compound 4 (solid). 


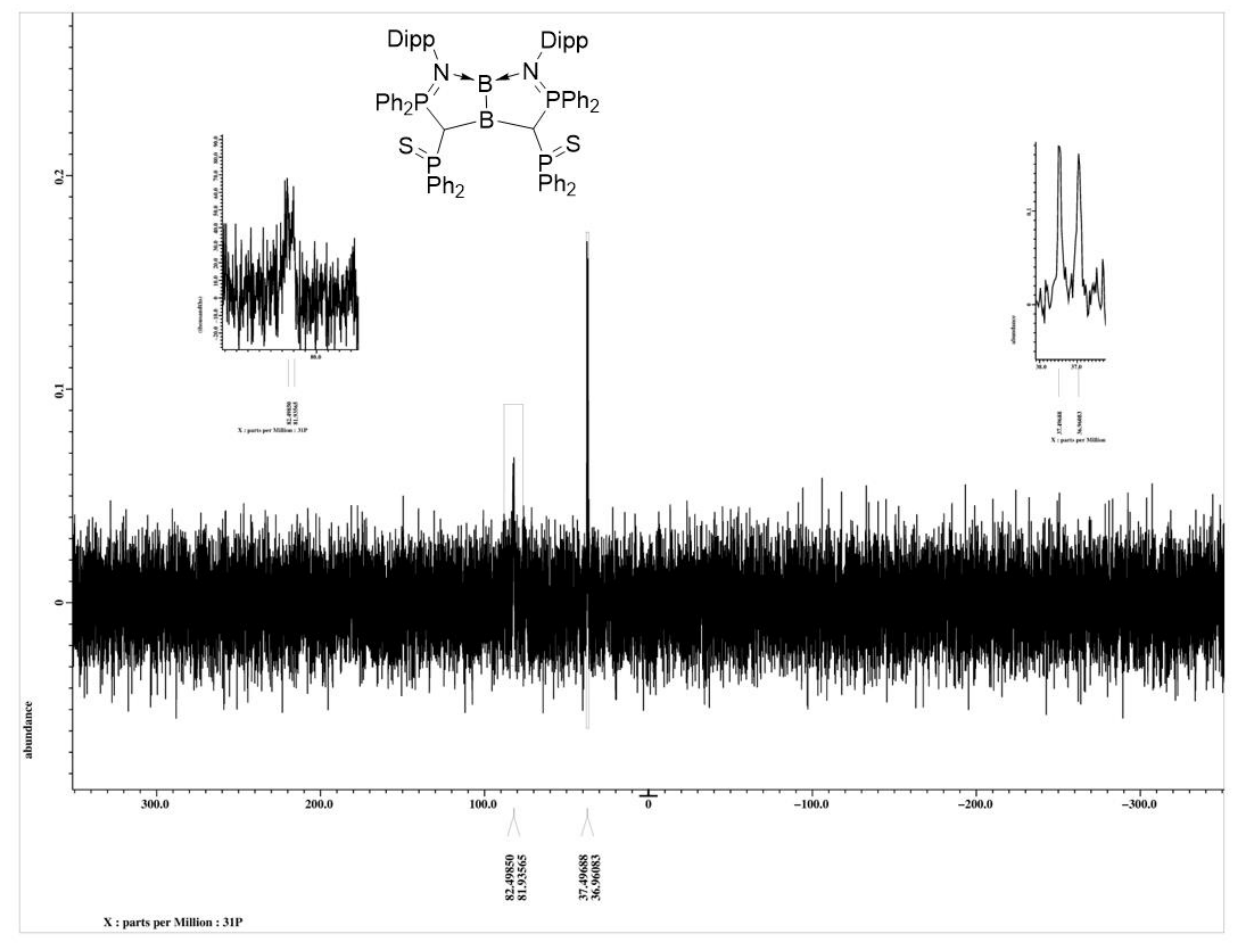

(a)

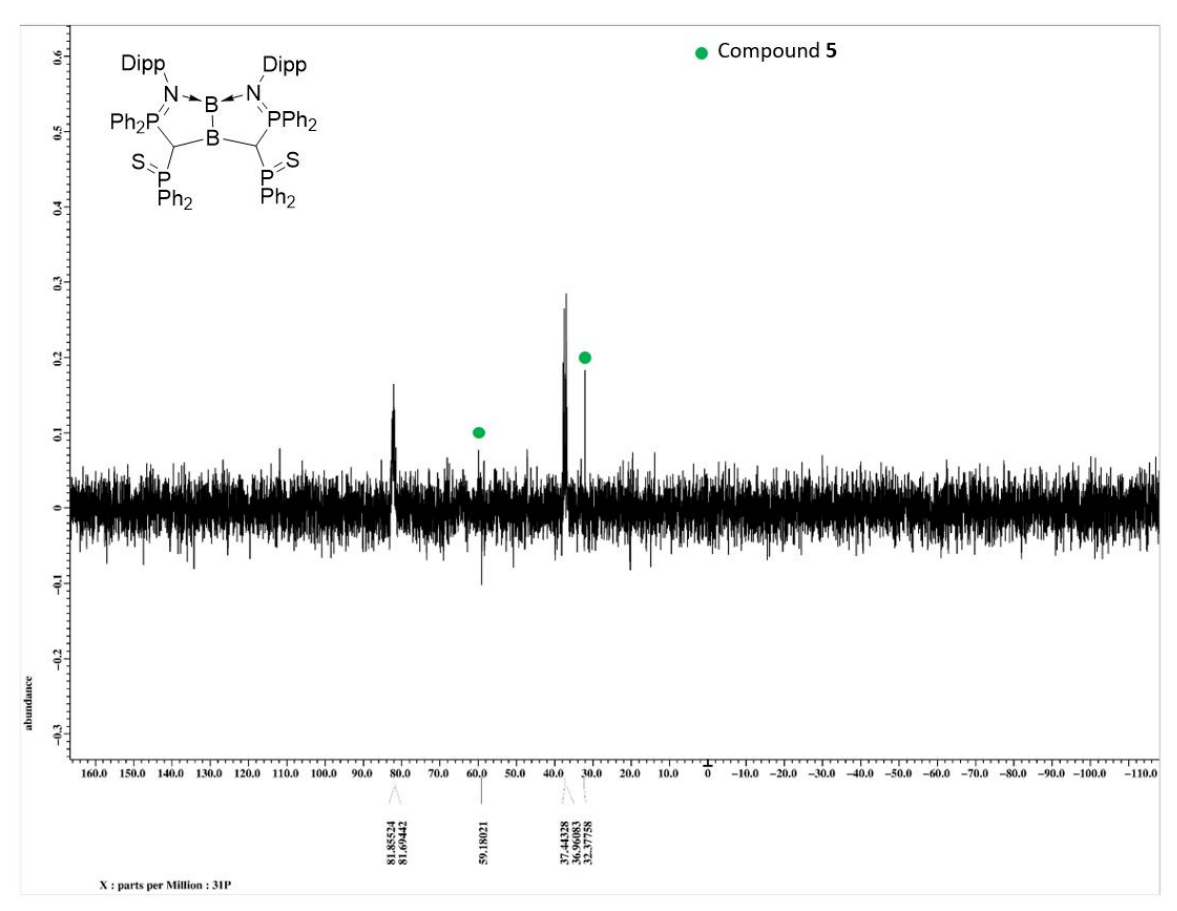

(b)

Figure S13. ${ }^{31} \mathrm{P}\left\{{ }^{1} \mathrm{H}\right\}$ NMR spectrum of compound 4 (in THF-d $\mathrm{d}_{8}$ ), (a) acquisition time: $5 \mathrm{mim}$. (b) acquisition time: $15 \mathrm{~min}$ 


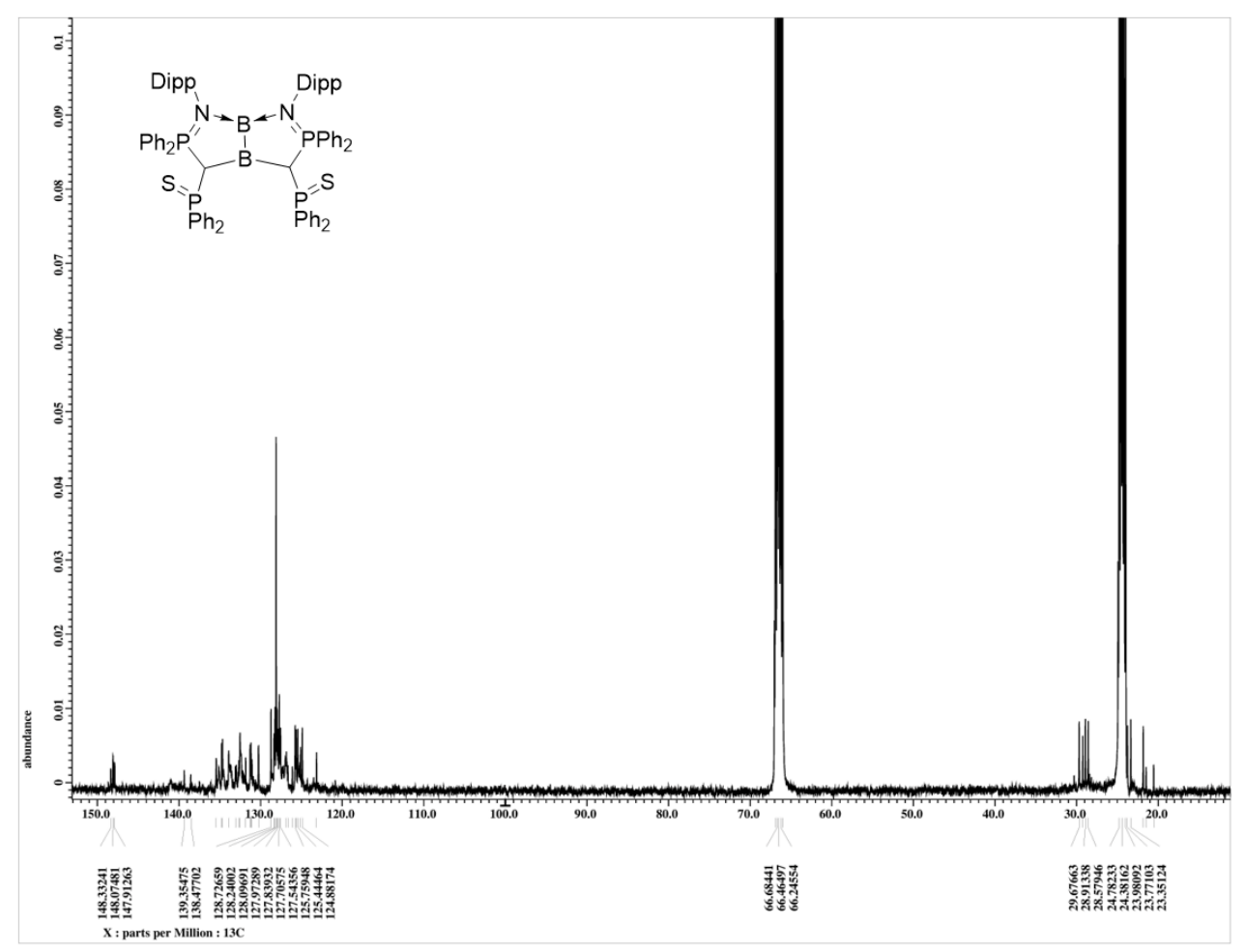

Figure S14. ${ }^{13} \mathrm{C}\left\{{ }^{1} \mathrm{H}\right\}$ NMR spectrum of compound 4 (in THF- $\mathrm{d}_{8}$ ).

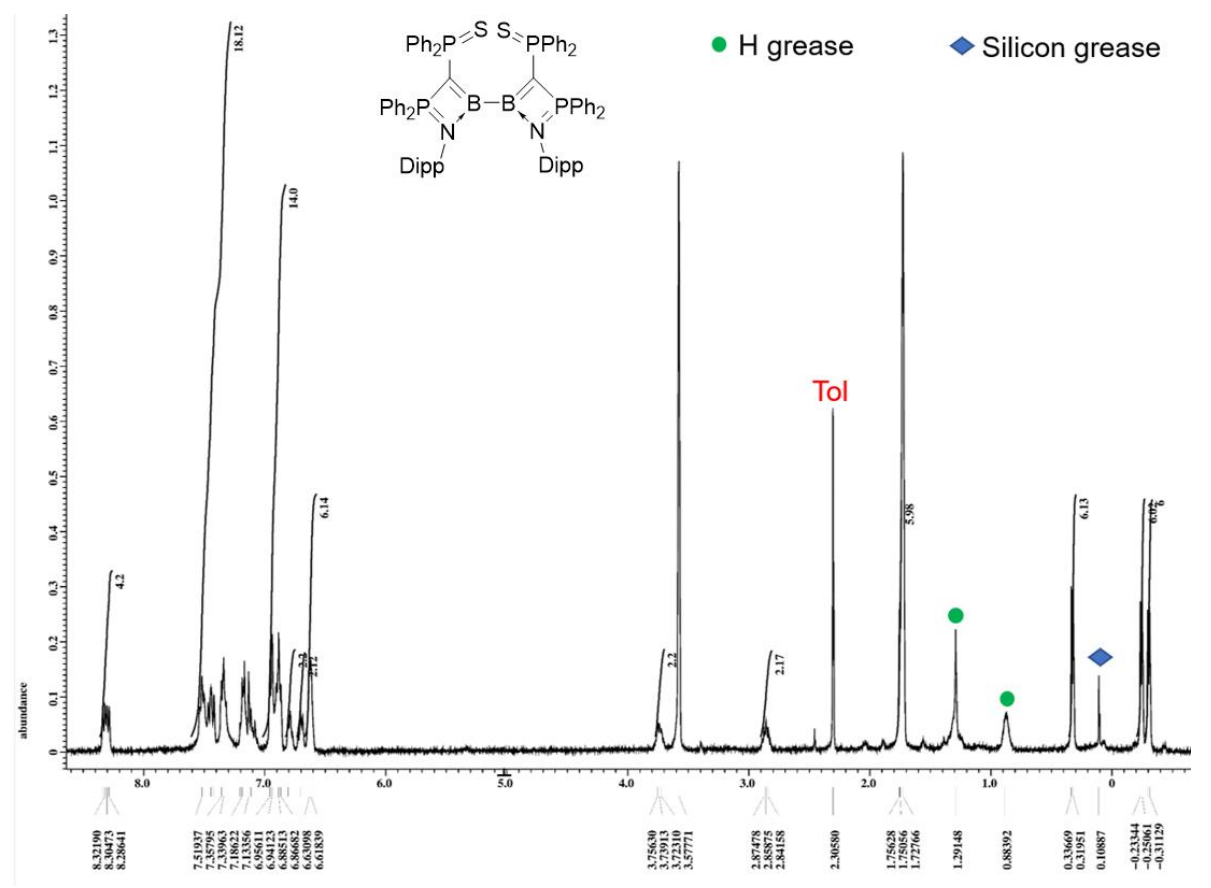

Figure S15. ${ }^{1} \mathrm{H}$ NMR spectrum of compound 5 (in THF-d $\mathrm{d}_{8}$ ). Signals at 0.3 and $0.9-1.3 \mathrm{ppm}$ correspond to silicone and $\mathrm{H}$ grease that were used to handle air- and moisture-sensitive compounds. 


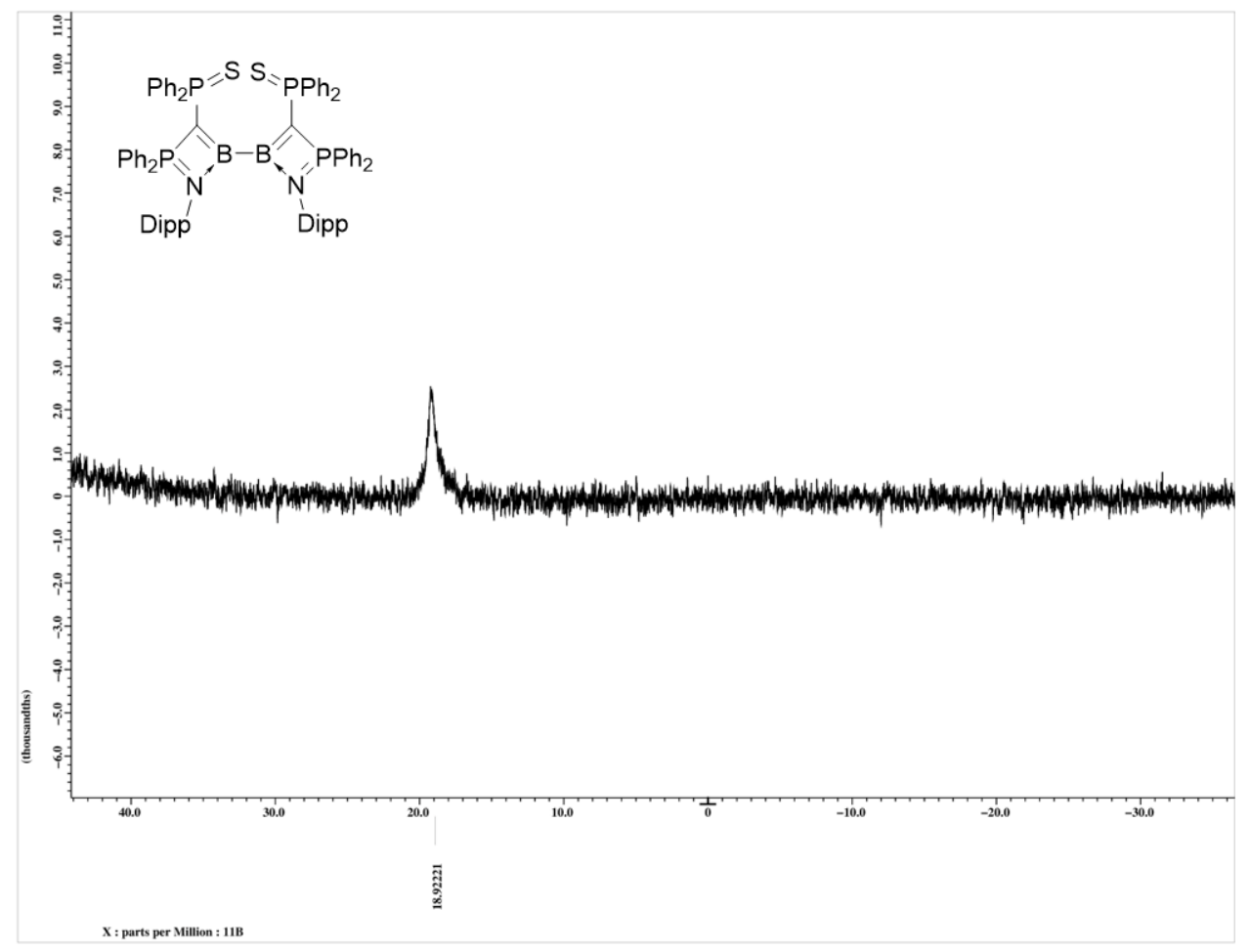

Figure S16. ${ }^{11} \mathrm{~B}\left\{{ }^{1} \mathrm{H}\right\}$ NMR spectrum of compound 5 (in THF-d $\mathrm{d}_{8}$ in quartz NMR tube).

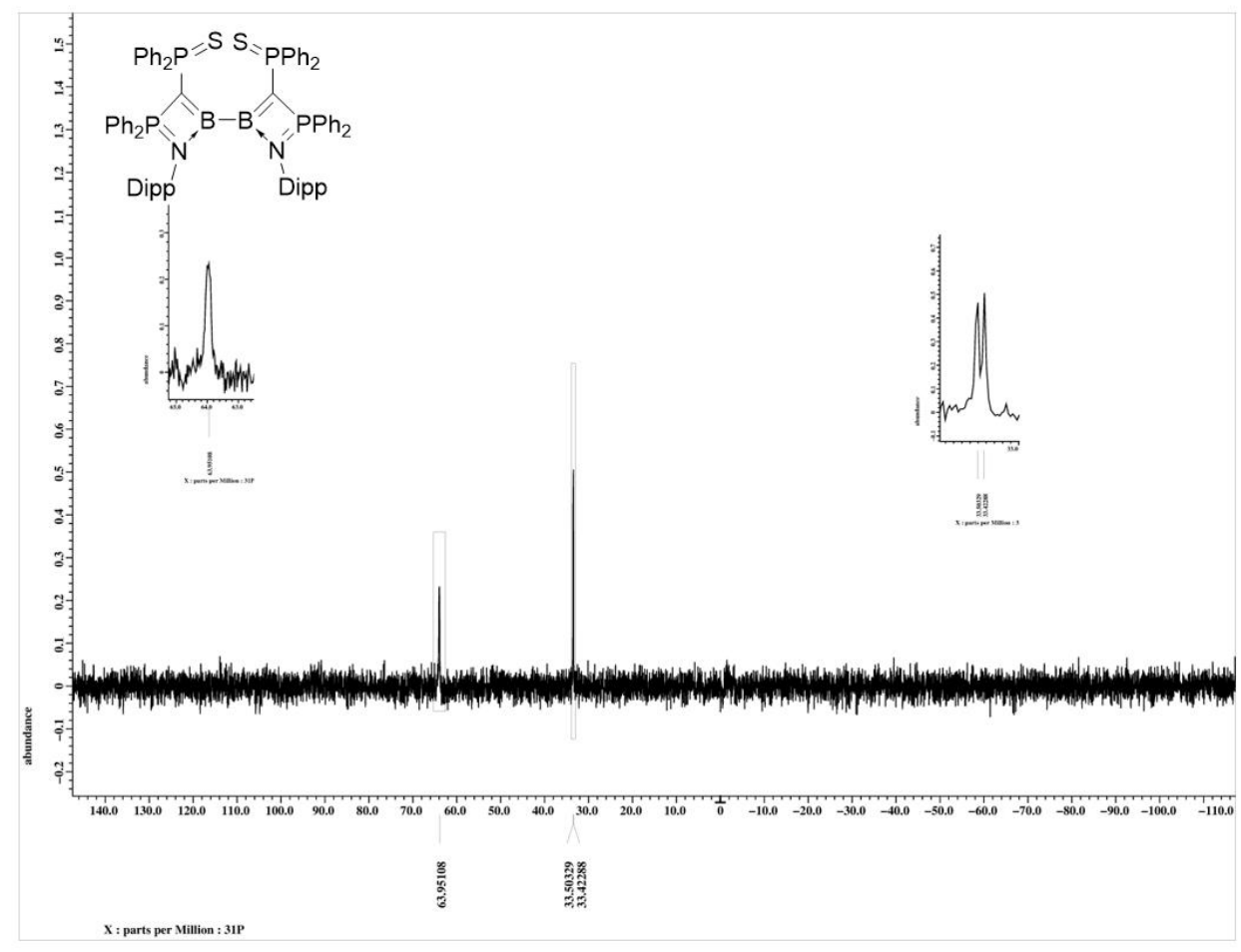

Figure S17. ${ }^{31} \mathrm{P}\left\{{ }^{1} \mathrm{H}\right\}$ NMR spectrum of compound 5 (in THF- $\mathrm{d}_{8}$ ). 


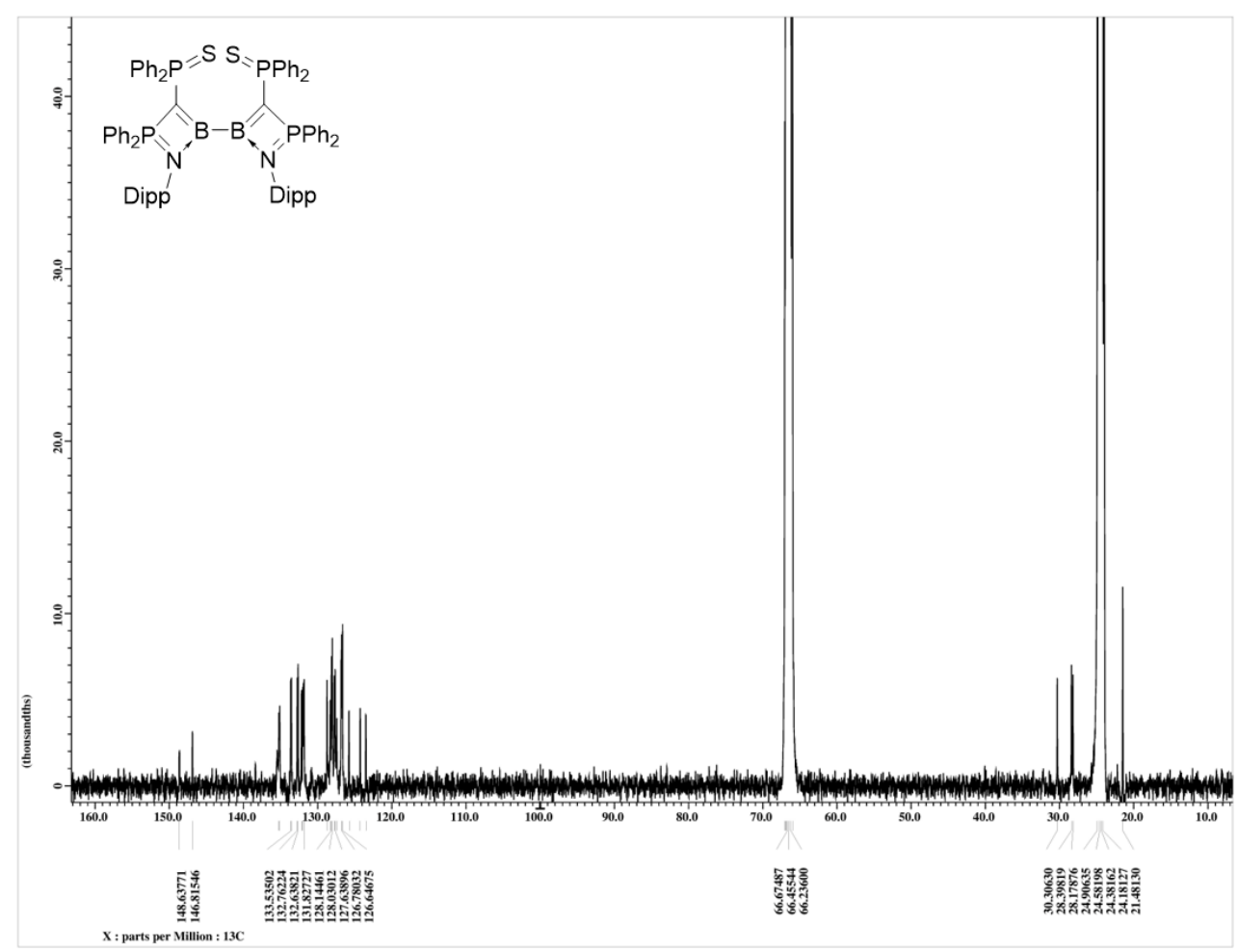

Figure S18. ${ }^{13} \mathrm{C}\left\{{ }^{1} \mathrm{H}\right\}$ NMR spectrum of compound 5 (in THF-d 8 ).

Elemental Composition Report

Page 1

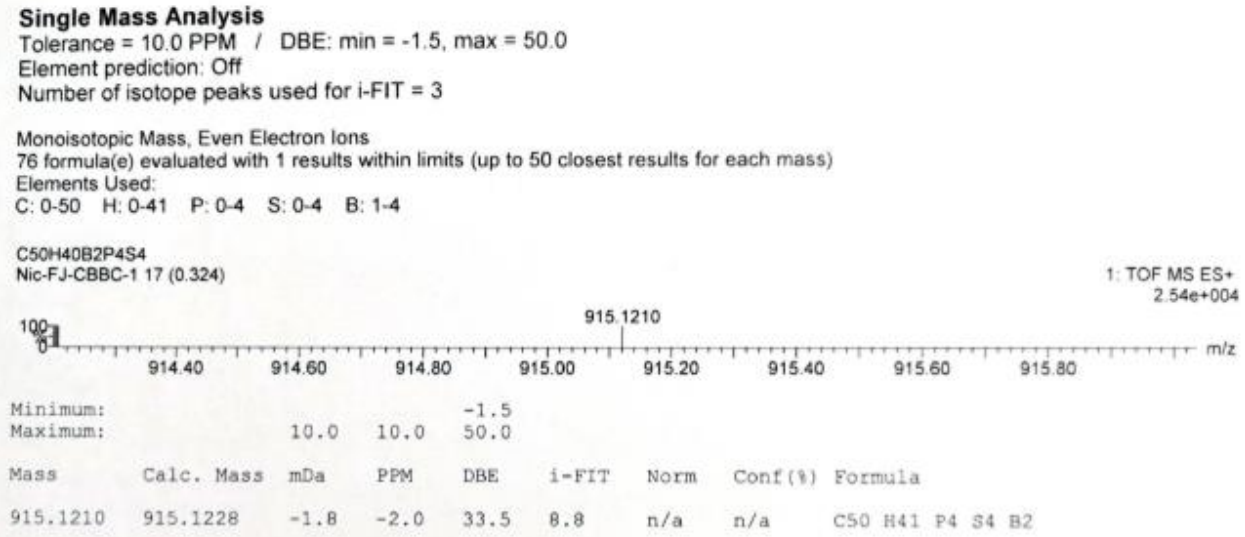

Figure S19. HRMS of compound 2. 
Single Mass Analysis

Tolerance $=10.0$ PPM $/$ DBE: $\min =-1.5, \max =50.0$
Element prediction: Off

Number of isotope peaks used for $\mathrm{i}-\mathrm{FIT}=3$

Monoisotopic Mass, Even Electron lons

18 formula(e) evaluated with 1 results within limits (up to 50 closest results for each mass)

Elements Used:

$\begin{array}{lllll}\text { C: } 0-37 & \text { H: } 0-40 & \text { N: }: 0-5 & \text { P: } 0-2 & \text { S: } 1-1\end{array}$

Nic-FJ-Ligand $113(2.027)$

1: TOF MS ES+
$2.23 \mathrm{e}+007$

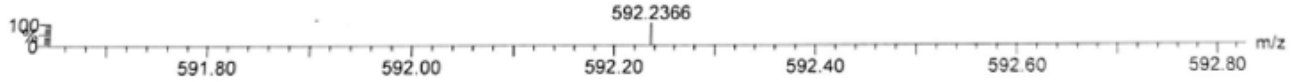

Minimum:

Maximum: $10.0 \quad 10.0 \quad 50.0$

Mass Calc. Mass mDa PPM DBE i-FIT Norm Conf(8) Formula

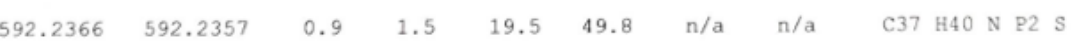

Figure S20. HRMS of $\mathrm{H}_{2} \mathrm{C}\left(\mathrm{PPh}_{2} \mathrm{NAr}\right)\left(\mathrm{PPh}_{2} \mathrm{~S}\right)$.

\section{Single Mass Analysis}

Tolerance $=10.0$ PPM $/$ DBE: $\min =-1.5, \max =50.0$

Element prediction: Off

Number of isotope peaks used for $\mathrm{i}-\mathrm{FIT}=3$

Monoisotopic Mass, Even Electron lons

34 formula(e) evaluated with 1 results within limits (up to 50 closest results for each mass)

Elements Used:

$\begin{array}{llllll}\text { C: } 0-37 & \text { H: } 0-38 & \text { Li: } 0-2 & \text { N: }: 0-2 & \text { P: } 0-2 & \text { S: } 0-1\end{array}$

Nic-FJ-Dianion $2(0.070)$

$\log _{7}$
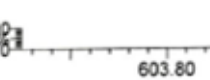

Figure S21. HRMS of compound 3. 


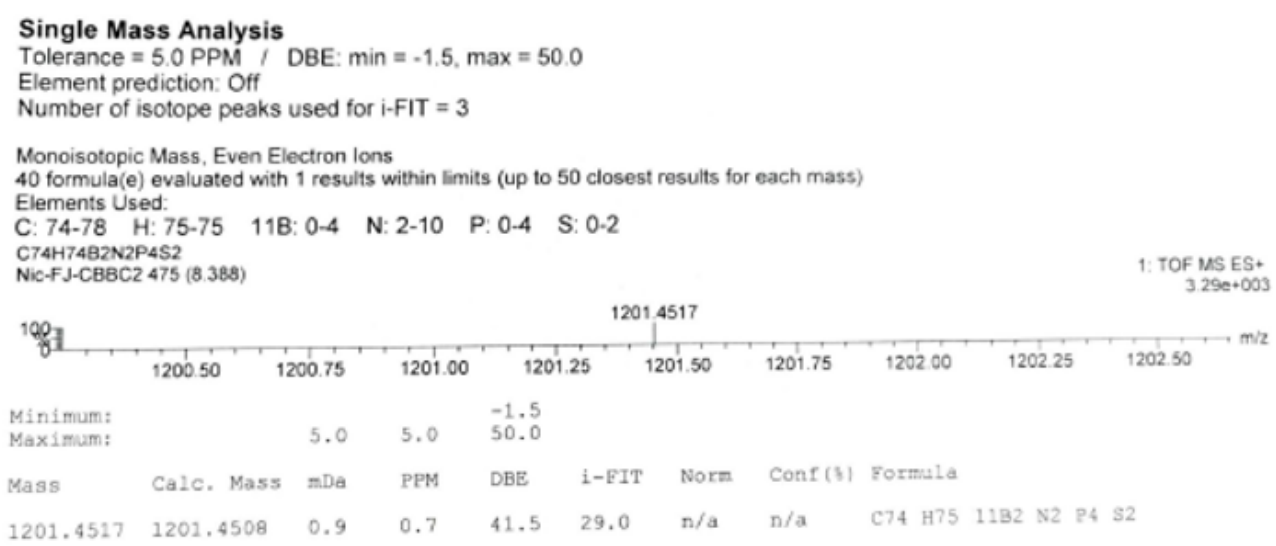

Figure S22. HRMS of compound 4.

\section{Elemental Composition Report}

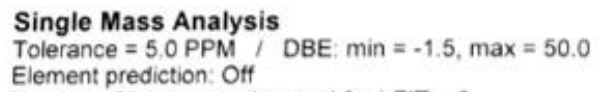

Figure S23. HRMS of compound 5. 


\section{S2. X-ray Data Collection and Structural Refinement}

X-ray data collection and structural refinement. The X-ray diffraction intensity data were measured at $100 \mathrm{~K}$ with a Bruker APEX II diffractometer equipped with a CCD detector, employing Mo $\mathrm{K} \alpha$ radiation $(\lambda=0.71073 \AA)$, with the SMART suite of programs. ${ }^{1}$ All data were processed and corrected for Lorentz and polarization effects with SAINT and for absorption effects with SADABS. Structural solution and refinement were carried out with the SHELXTL suite of programs. ${ }^{2}$ The structures were solved by direct methods to locate the heavy atoms, followed by difference maps for the light, non-hydrogen atoms. All non-hydrogen atoms were refined with anisotropic thermal parameters. The X-ray crystal structure of $\mathbf{2}$ has a whole molecule disorder (94:6). CCDC-2051547 2, CCDC-2079079 [ $\left.\mathrm{H}_{2} \mathrm{C}\left(\mathrm{PPh}_{2} \mathrm{~S}\right)\left(\mathrm{PPh}_{2} \mathrm{NAr}\right)\right]$, CCDC-2051548 4 and CCDC-2051549 5 contain the supplementary crystallographic data for this paper. The data can be obtained free of charge from the Cambridge Crystallography Data Center via www.ccdc.cam.ac.uk/data request/cif.

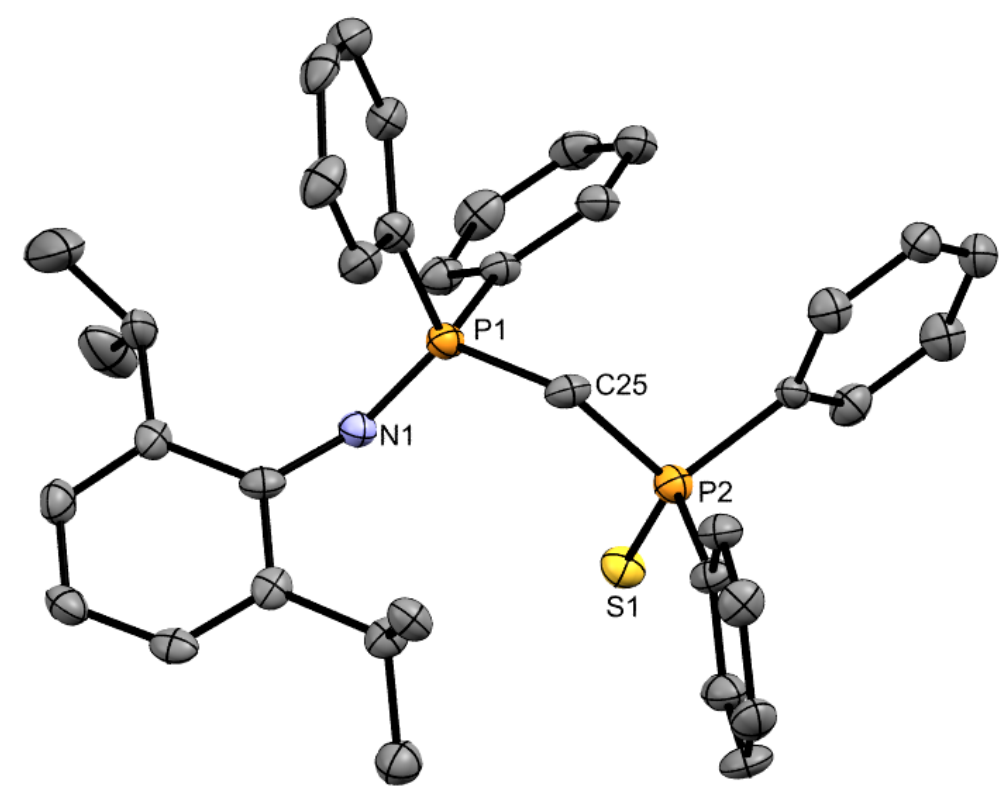

Figure S24. X-ray crystal structure of $\left[\mathrm{H}_{2} \mathrm{C}\left(\mathrm{PPh}_{2} \mathrm{~S}\right)\left(\mathrm{PPh}_{2} \mathrm{NAr}\right)\right]$. with thermal ellipsoids at 50 $\%$ probability. All $\mathrm{H}$ atoms are omitted for clarity. Selected bond lengths ( $\mathrm{A})$ and angles (deg): P1-N1 1.537(6), P1-C25 1.831(6), P2-C25 1.844(6), P2-S1 1.946(3), P1-C25-P2 120.3(3), C25-P1-N1 117.6(3), C25-P2-S1 116.5(2). 
Table S1. X-Ray crystallographic data for compounds $2,4,5$ and $\mathrm{H}_{2} \mathrm{C}\left(\mathrm{PPh}_{2} \mathrm{NAr}\right)\left(\mathrm{PPh}_{2} \mathrm{~S}\right)$.

\begin{tabular}{|c|c|c|c|c|}
\hline & $\mathbf{2} \cdot(\text { Fluorobenzene })_{2}$ & $\mathbf{4} \cdot(\text { Toluene })_{2}$ & $\mathbf{5} \cdot(\text { Toluene })_{1.5}$ & $\mathbf{H}_{2} \mathbf{C}\left(\mathbf{P P h}_{2} \mathbf{N A r}\right)\left(\mathbf{P P h}_{2} \mathrm{~S}\right)$ \\
\hline Formula & $\mathrm{C}_{62} \mathrm{H}_{50} \mathrm{~B}_{2} \mathrm{~F}_{2} \mathrm{P}_{4} \mathrm{~S}_{4}$ & $\mathrm{C}_{95} \mathrm{H}_{99} \mathrm{~B}_{2} \mathrm{~N}_{2} \mathrm{P}_{4} \mathrm{~S}_{2}$ & $\mathrm{C}_{84.50} \mathrm{H}_{86} \mathrm{~B}_{2} \mathrm{~N}_{2} \mathrm{P}_{4} \mathrm{~S}_{2}$ & $\mathrm{C}_{37} \mathrm{H}_{39} \mathrm{NP}_{2} \mathrm{~S}$ \\
\hline Fw & 1106.76 & 1478.38 & 1339.17 & 591.69 \\
\hline$T / \mathrm{K}$ & $100(2)$ & $100(2)$ & $100(2)$ & $100(2)$ \\
\hline cryst system & triclinic & monoclinic & triclinic & triclinic \\
\hline space group & $P-1$ & $P 121 / c 1$ & $P-1$ & $P-1$ \\
\hline$a(\AA)$ & $9.0566(5)$ & $12.7608(3)$ & $17.2565(6)$ & $8.9627(8)$ \\
\hline$b(\AA)$ & $12.6636(8)$ & $21.9403(6)$ & $20.1879(7)$ & $11.9406(10)$ \\
\hline$c(\AA)$ & $13.8660(8)$ & $28.9608(6)$ & $21.0330(7)$ & $16.4349(13)$ \\
\hline$\alpha(\mathrm{deg})$ & $63.8065(19)$ & 90 & $84.2902(11)$ & $78.653(2)$ \\
\hline$\beta(\mathrm{deg})$ & 87.0006 & $97.9810(10)$ & $85.1174(12)$ & $74.639(2)$ \\
\hline$\gamma(\operatorname{deg})$ & $74.813(2)$ & 90 & $85.0595(11)$ & $72.022(2)$ \\
\hline$V\left(\AA^{3}\right)$ & $1373.45(14)$ & $8029.8(3)$ & $7242.2(4)$ & $1600.5(2)$ \\
\hline$Z$ & 1 & 4 & 4 & 2 \\
\hline$d_{\text {calcd }}\left(\mathrm{g} \mathrm{cm}^{-3}\right)$ & 1.338 & 1.223 & 1.228 & 1.228 \\
\hline$\mu\left(\mathrm{mm}^{-1}\right)$ & 0.337 & 0.195 & 0.209 & 0.228 \\
\hline$F(000)$ & 574 & 3140 & 2836 & 628 \\
\hline cryst size (mm) & $\begin{array}{l}0.100 \times 0.120 \times \\
0.260\end{array}$ & $\begin{array}{l}0.080 \times 0.120 \times \\
0.200\end{array}$ & $\begin{array}{l}0.120 \times 0.200 \times \\
0.220\end{array}$ & $0.010 \times 0.018 \times 0.080$ \\
\hline $2 \theta$ range $(\mathrm{deg})$ & $4.672<2 \theta<52.06$ & $\begin{array}{l}4.992<2 \theta \\
<59.08\end{array}$ & $2.27<2 \theta<29.00$ & $4.704<2 \theta<49.85$ \\
\hline index range & $\begin{array}{l}-13<=h<=13, \\
-18<=k<=18, \\
-20<=l<=20\end{array}$ & $\begin{array}{l}-17<=h<=16, \\
-24<=k<=30, \\
-38<=k<=40\end{array}$ & $\begin{array}{l}-23<=h<=23, \\
-27<=k<=27, \\
-28<=l<=28\end{array}$ & $\begin{array}{l}-10<=\mathrm{h}<=10, \\
14<=\mathrm{k}<=14, \\
-19<=\mathrm{l}<=18\end{array}$ \\
\hline $\begin{array}{l}\text { no. of rflns } \\
\text { collected }\end{array}$ & 53887 & 73804 & 167754 & 19935 \\
\hline no. of indep rflns & 8809 & 22466 & 38220 & 5649 \\
\hline $\begin{array}{l}R 1, w R 2(I> \\
2 \sigma(I))\end{array}$ & 0.0690 & 0.0473 & 0.0588 & 0.0775 \\
\hline$R 1, w R 2$ (all data) & 0.1551 & 0.0731 & 0.1218 & 0.1816 \\
\hline goodness of fit, $F^{2}$ & 1.013 & 1.03 & 1.017 & 1.022 \\
\hline $\begin{array}{l}\text { no. of } \\
\text { data/restraints/par } \\
\text { ams }\end{array}$ & 8809 / $2005 / 562$ & $\begin{array}{l}22466 / 612 / \\
1062\end{array}$ & $\begin{array}{l}38220 / 894 / \\
1822\end{array}$ & $5649 / 0 / 374$ \\
\hline $\begin{array}{l}\text { largest diff peak } \\
\text { and hole, } \mathrm{e}^{-3}\end{array}$ & $0.444 /-0.578$ & $0.649 /-0.924$ & $0.607 /-0.531$ & $0.650 /-0.460$ \\
\hline
\end{tabular}




\section{S3. Theoretical Studies}

All geometry optimizations were performed without any symmetry restriction by using the M06-2X functional with def2-SVP basis set as implemented in the Gaussian 09 package of programs. ${ }^{3,4}$ The vibrational frequency calculations were performed to establish the nature of stationary points. The local minima were confirmed by zero imaginary frequencies. The NBO analyses were performed using the NBO 6.0 program at the same level. ${ }^{5}$ The optimized geometries are in good agreement with their X-ray crystallographic data.

List of colour coded atom used in optimised structures

\begin{tabular}{|c|c|}
\hline Abbreviation & Definition \\
\hline Grey & Carbon \\
Blue & Nitrogen \\
Yellow & Sulfur \\
Pink & Boron \\
Orange & Phosphorus \\
\hline
\end{tabular}




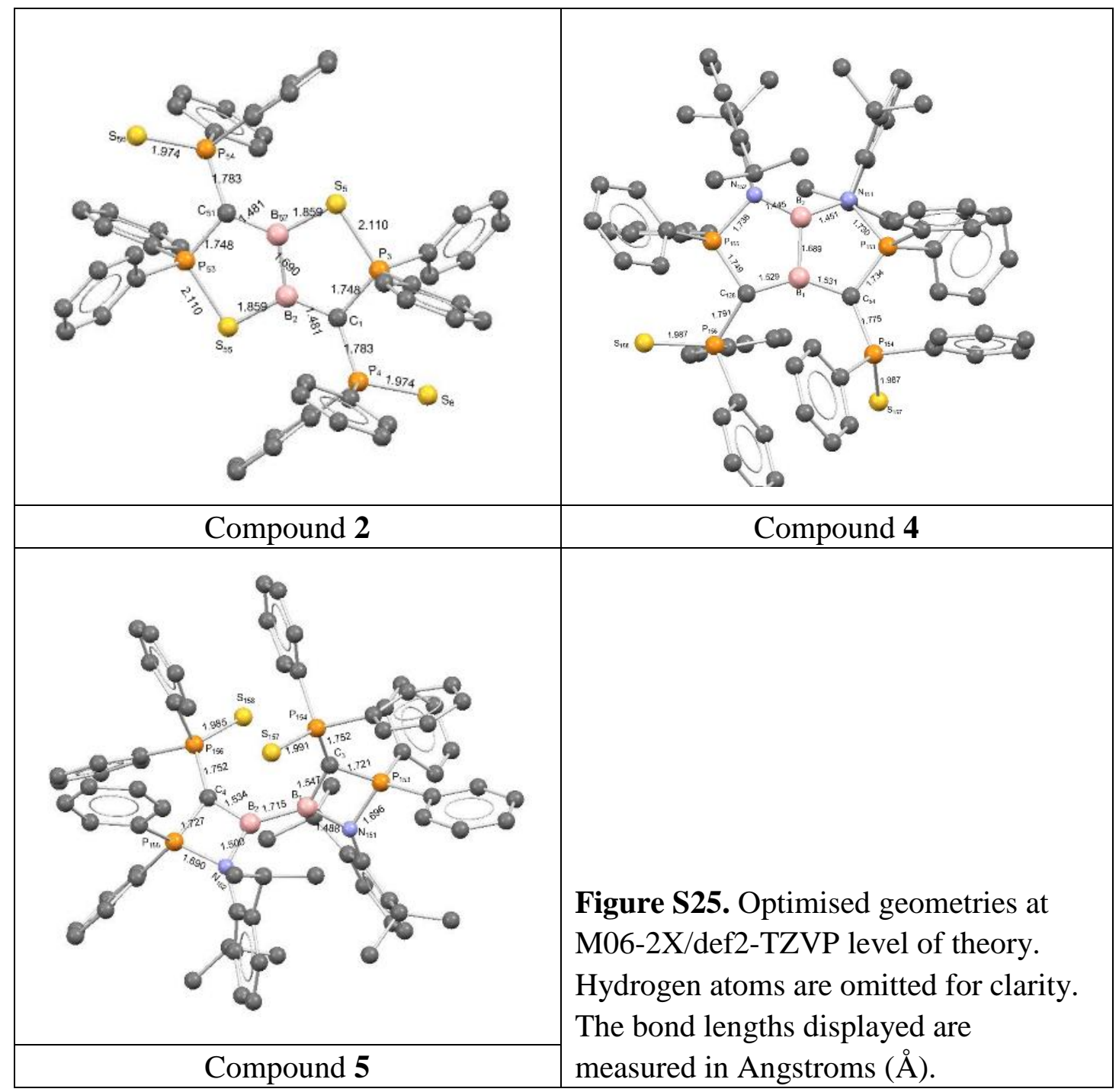

Table S2. Optimized geometric parameters and Wiberg Bond Index of compound 2 at M06-2X/def2-SVP.

\begin{tabular}{ccc}
\hline Bond No. & Bond Distance $(\AA)$ & Wiberg Bond Index \\
\hline $1($ C-B $)$ & 1.481 & 1.224 \\
$2(\mathrm{C}-\mathrm{P})$ & 1.748 & 1.010 \\
$3(\mathrm{P}-\mathrm{S})$ & 2.110 & 0.982 \\
$4(\mathrm{~S}-\mathrm{B})$ & 1.859 & 1.080 \\
$5(\mathrm{~B}-\mathrm{B})$ & 1.690 & 0.967 \\
6(C-B) & 1.481 & 1.223 \\
$7(\mathrm{C}-\mathrm{P})$ & 1.748 & 1.010 \\
$8(\mathrm{P}-\mathrm{S})$ & 2.110 & 0.982
\end{tabular}




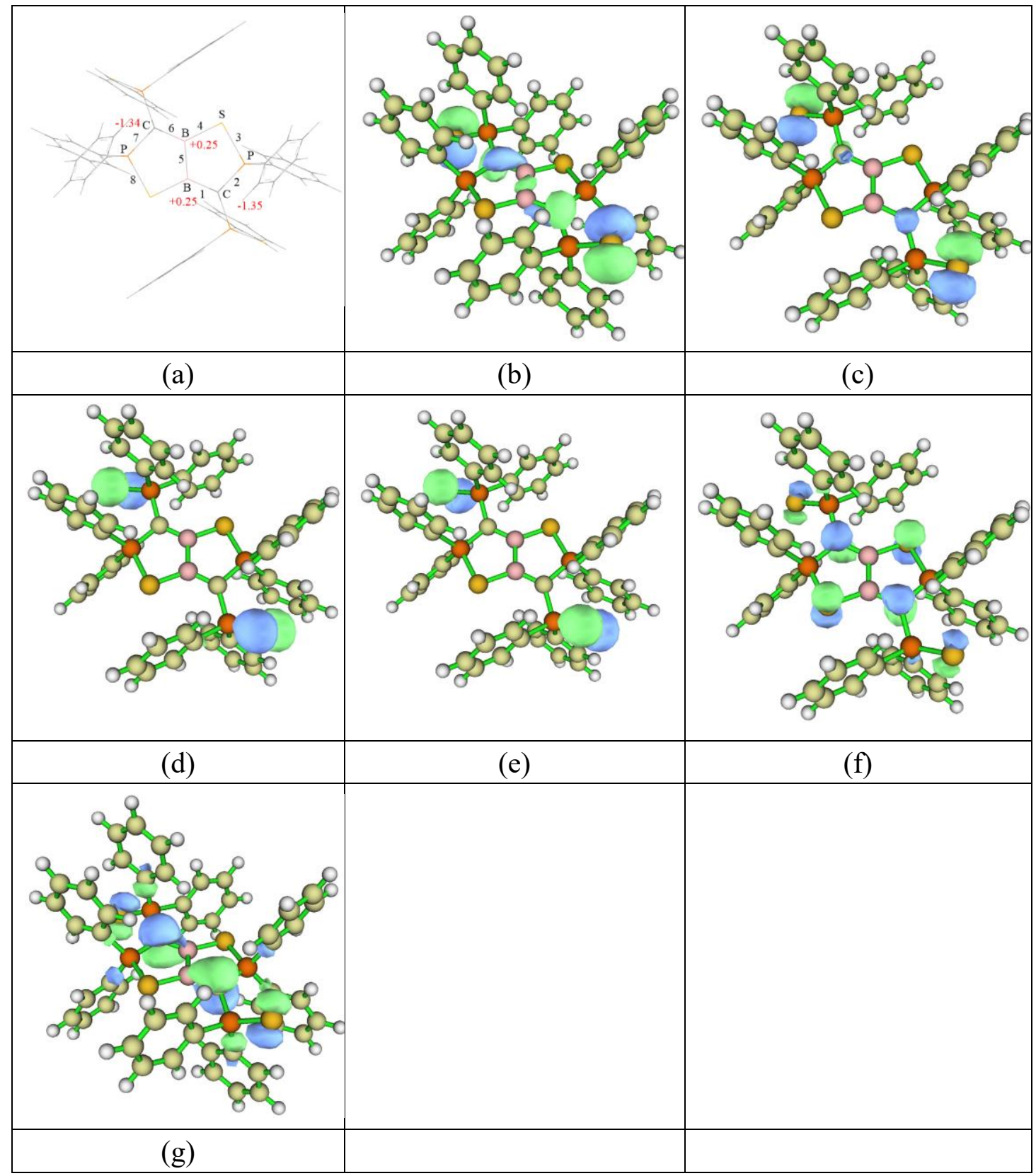

Figure S26. (a) Natural charge population of compound 2. Natural charges on C, B were shown in red. (b) HOMO (-6.72 eV). (c) HOMO-1 (-6.91 eV). (d) HOMO-2 ($7.03 \mathrm{eV}$ ). (e) HOMO-3 (-7.03 eV). (f) HOMO-4 (-7.34 eV). (g) HOMO-5 (-7.42 eV). B: pink, P: orange, S: dark yellow, C: yellow, H: white. 
Table S3. NBO analysis of compound 2.

\begin{tabular}{|c|c|c|c|c|}
\hline & Orbital & Occupancy & Contribution & Atomic Orbitals \\
\hline $1(\mathrm{C}-\mathrm{B}, \sigma)$ & & 1.95 & $\begin{array}{l}70.08 \% \mathrm{C}+ \\
29.92 \% \mathrm{~B}\end{array}$ & $\begin{array}{l}\mathrm{C}: 46.10 \% \mathrm{~s}+ \\
53.96 \% \mathrm{p} \\
\mathrm{B}: 36.96 \% \mathrm{~s}+ \\
62.92 \% \mathrm{p}\end{array}$ \\
\hline $1(\mathrm{C}-\mathrm{B}, \pi)$ & & 1.82 & $\begin{array}{l}80.68 \% \mathrm{C}+ \\
19.32 \% \mathrm{~B}\end{array}$ & $\begin{array}{l}\text { C: } 99.93 \% p \\
\text { B: } 99.86 \% p\end{array}$ \\
\hline $2(\mathrm{C}-\mathrm{P}, \sigma)$ & & 1.96 & $\begin{array}{l}59.36 \% \mathrm{C}+ \\
40.64 \% \mathrm{P}\end{array}$ & $\begin{array}{l}\mathrm{C}: 24.29 \% \mathrm{~s}+ \\
75.68 \% \mathrm{p} \\
\mathrm{P}: 31.62 \% \mathrm{~s}+ \\
67.83 \% \mathrm{p}\end{array}$ \\
\hline $3(\mathrm{P}-\mathrm{S}, \sigma)$ & & 1.96 & $\begin{array}{l}45.31 \% \mathrm{P}+ \\
54.69 \% \mathrm{~S}\end{array}$ & $\begin{array}{l}\mathrm{P}: 18.89 \% \mathrm{~s}+ \\
79.81 \% \mathrm{p} \\
\mathrm{S}: 12.14 \% \mathrm{~s}+ \\
87.32 \% \mathrm{p}\end{array}$ \\
\hline $4(\mathrm{~S}-\mathrm{B}, \sigma)$ & & 1.97 & $\begin{array}{l}67.34 \% \mathrm{~S}+ \\
32.66 \% \mathrm{~B}\end{array}$ & $\begin{array}{l}\mathrm{S}: 32.45 \% \mathrm{~s}+ \\
67.33 \% \mathrm{p} \\
\mathrm{B}: 25.65 \% \mathrm{~s}+ \\
74.11 \% \mathrm{p}\end{array}$ \\
\hline $5(\mathrm{~B}-\mathrm{B}, \sigma)$ & & 1.93 & $\begin{array}{l}50.00 \% \mathrm{~B}+ \\
50.00 \% \mathrm{~B}\end{array}$ & $\begin{array}{l}\mathrm{B}: 38.48 \% \mathrm{~s}+ \\
61.51 \% \mathrm{p} \\
\mathrm{B}: 38.48 \% \mathrm{~s}+ \\
61.51 \% \mathrm{p}\end{array}$ \\
\hline
\end{tabular}




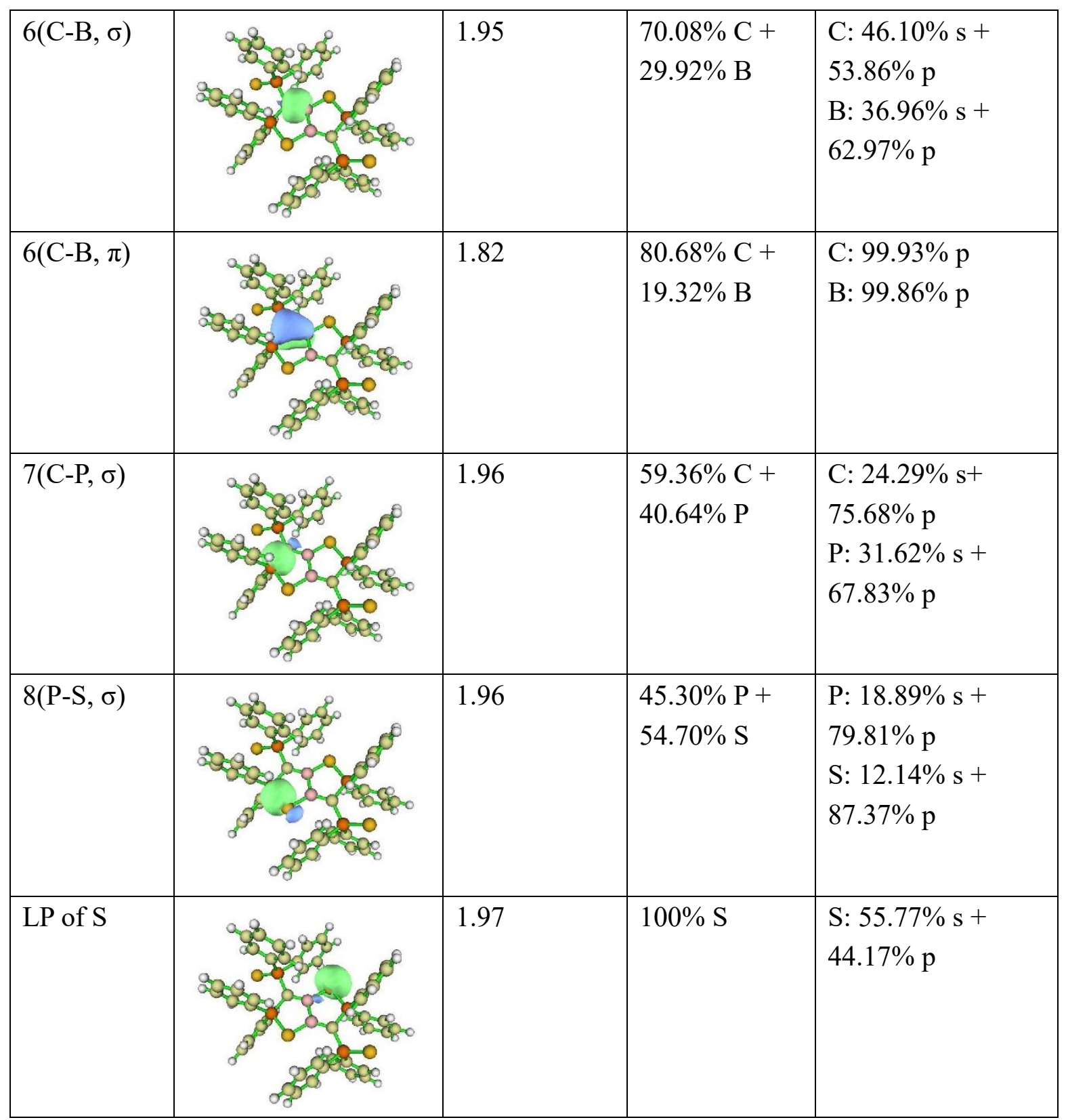


Table S4. Optimized geometric parameters and Wiberg Bond Index of compound 4 at M06-2X/def2-SVP.

\begin{tabular}{|c|c|c|}
\hline Bond No. & Bond Distance (Å) & Wiberg Bond Index \\
\hline $1(B-C)$ & 1.531 & 1.067 \\
\hline $2(C-P)$ & 1.734 & 1.068 \\
\hline $3(P-N)$ & 1.730 & 0.778 \\
\hline $4(B-N)$ & 1.451 & 0.849 \\
\hline $5(B-B)$ & 1.689 & 0.927 \\
\hline $6(B-N)$ & 1.445 & 0.867 \\
\hline $7(\mathrm{P}-\mathrm{N})$ & 1.738 & 0.759 \\
\hline $8(C-P)$ & 1.749 & 1.058 \\
\hline $9(C-B)$ & 1.529 & 1.097 \\
\hline
\end{tabular}




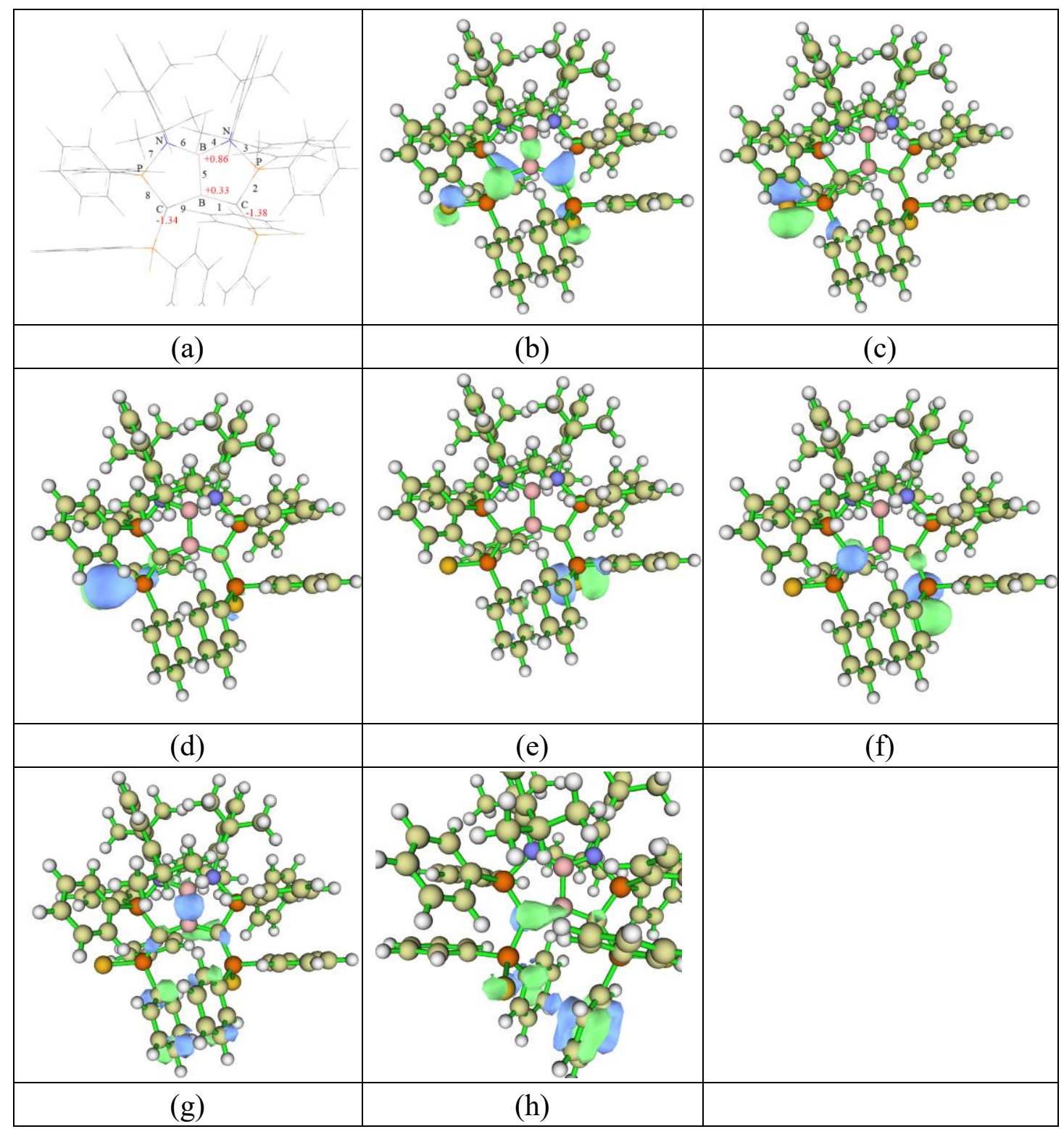

Figure S27. (a) Natural charge population of compound 4. Natural charges on C, B were shown in red. (b) HOMO(-6.05 eV). (c) HOMO-1(-6.42 eV). (d) HOMO-2 ($6.57 \mathrm{eV})$. (e) HOMO-3 (-6.64 eV). (f) HOMO-4 (-6.89 eV). (g) HOMO-5 (-7.50 eV). (h) HOMO-6 (-7.68 eV). B: pink, P: orange, S: dark yellow, C: yellow, H: white. 
Table S5. NBO analysis of compound 4.

\begin{tabular}{|c|c|c|c|c|}
\hline & Orbital & Occupancy & Contribution & Atomic Orbitals \\
\hline $1(\mathrm{~B}-\mathrm{C}, \sigma)$ & & 1.93 & $\begin{array}{l}29.80 \% \mathrm{~B}+ \\
70.20 \% \mathrm{C}\end{array}$ & $\begin{array}{l}\text { B: } 35.07 \% \mathrm{~s}+ \\
64.87 \% \mathrm{p} \\
\mathrm{C}: 44.04 \% \mathrm{~s}+ \\
55.93 \% \mathrm{p}\end{array}$ \\
\hline $2(\mathrm{C}-\mathrm{P}, \sigma)$ & & 1.96 & $\begin{array}{l}59.31 \% \mathrm{C}+ \\
40.69 \% \mathrm{P}\end{array}$ & $\begin{array}{l}\mathrm{C}: 26.48 \% \mathrm{~s}+ \\
73.42 \% \mathrm{p} \\
\mathrm{P}: 30.07 \% \mathrm{~s}+ \\
67.27 \% \mathrm{p}\end{array}$ \\
\hline $2(\mathrm{C}-\mathrm{P}, \pi)$ & & 1.64 & $\begin{array}{l}93.97 \% \mathrm{C}+ \\
6.03 \% \mathrm{P}\end{array}$ & $\begin{array}{l}\text { C: } 99.94 \% p \\
\text { P: } 49.88 \% p+ \\
50.05 \% \mathrm{~d}\end{array}$ \\
\hline $3(\mathrm{P}-\mathrm{N}, \sigma)$ & & 1.93 & $\begin{array}{l}27.65 \% \mathrm{P}+ \\
72.35 \% \mathrm{~N}\end{array}$ & $\begin{array}{l}\text { P: } 20.88 \% \mathrm{~s}+ \\
78.03 \% \mathrm{p} \\
\mathrm{N}: 38.98 \% \mathrm{~s}+ \\
61.00 \% \mathrm{p}\end{array}$ \\
\hline $4(\mathrm{~B}-\mathrm{N}, \sigma)$ & & 1.96 & $\begin{array}{l}19.72 \% \mathrm{~B}+ \\
80.28 \% \mathrm{~N}\end{array}$ & $\begin{array}{l}\text { B: } 30.25 \% \mathrm{~s}+ \\
69.52 \% \mathrm{p} \\
\mathrm{N}: 42.41 \% \mathrm{~s}+ \\
57.56 \% \mathrm{p}\end{array}$ \\
\hline $5(\mathrm{~B}-\mathrm{B}, \sigma)$ & & 1.89 & $\begin{array}{l}51.15 \% \mathrm{~B}+ \\
48.85 \% \mathrm{~B}\end{array}$ & $\begin{array}{l}\text { B: } 31.26 \% \mathrm{~s}+ \\
68.72 \% \mathrm{p} \\
\mathrm{B}: 35.09 \% \mathrm{~s}+ \\
64.90 \% \mathrm{p}\end{array}$ \\
\hline
\end{tabular}




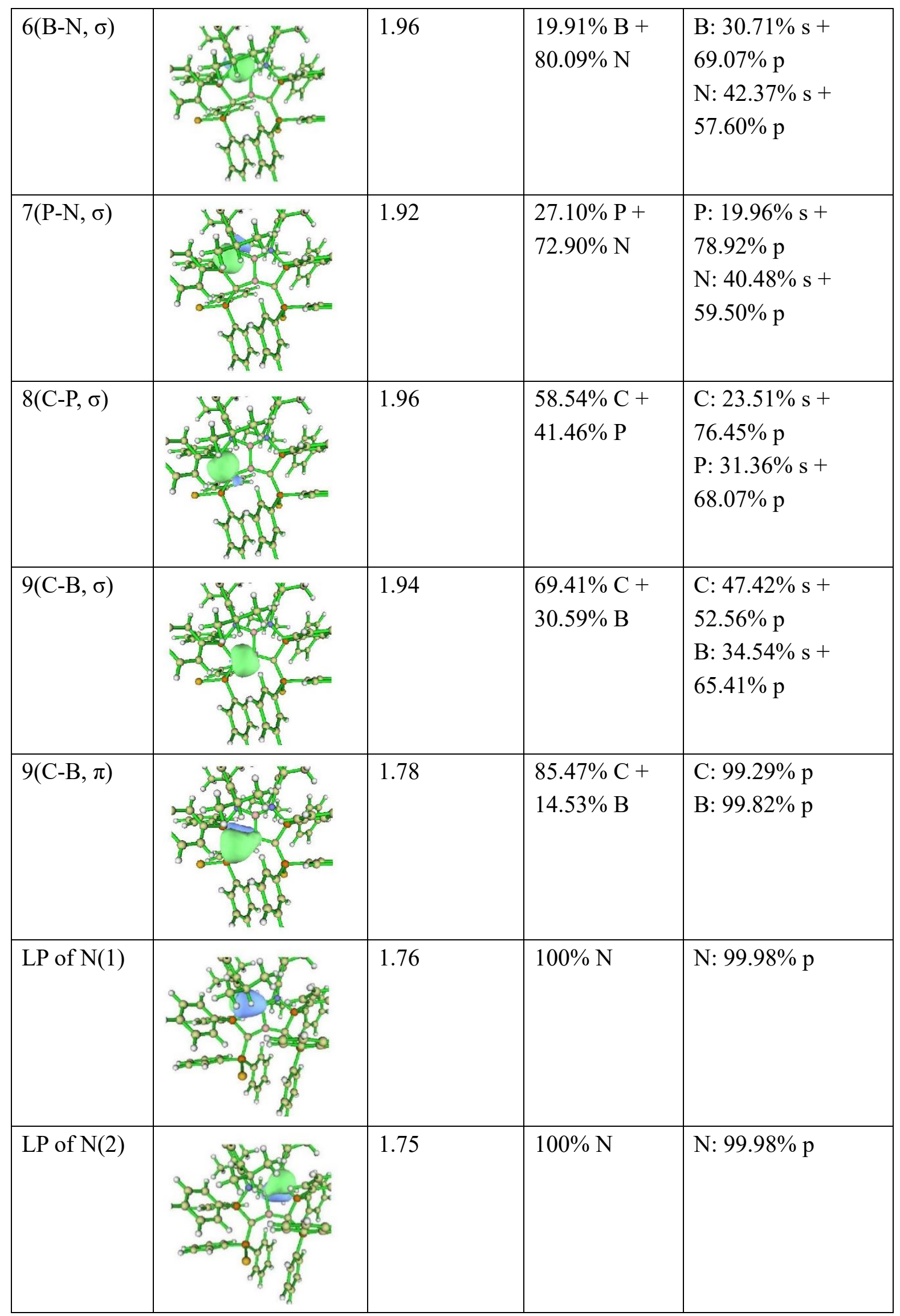


The NBO analysis of compound $\mathbf{4}$ shows that there is a geminal dianion coordinate to the boron center of the $\mathrm{C}=\mathrm{B}$ double bond. One lone pair of the geminal dianion coordinates with the boron center, while the other one is stabilized by the negative hyperconjugation with the P-C and P-N $\sigma^{*}$ orbital, leading to the C-P $\pi$ orbital in the NBO analysis. The C-P $\pi$ orbital is highly polarized toward the $\mathrm{C}$ atom (94.0\%), indicating that the negative hyperconjugation is weak. By checking the molecular orbitals, two molecular orbitals involving C-P-N were found, however, it was found that their electron occupation number were very small ( $\sim 0.08)$, implying that the effect of negative hyperconjugation should be very small.

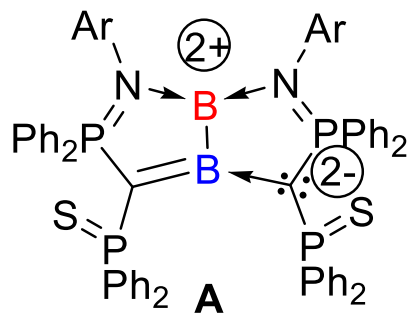
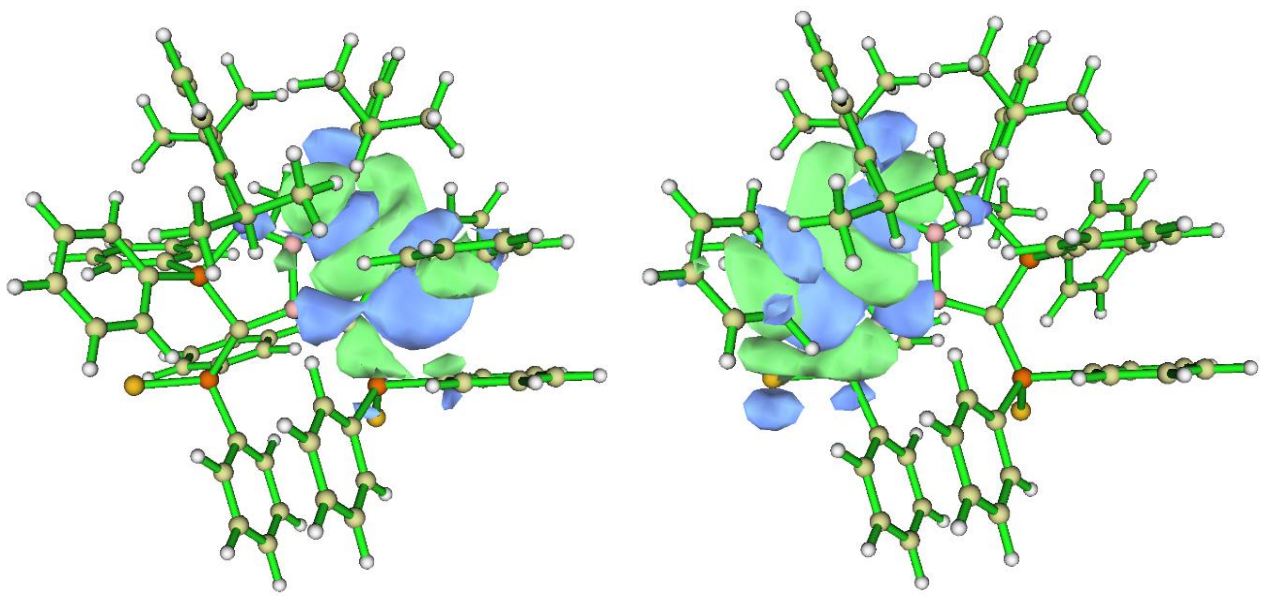

Figure S28. Molecular orbitals at the P-C-P skeleton of compound 4 (isovalue $=0.01$ ) 
Table S6. Optimized geometric parameters and Wiberg Bond Index of compound 5 at M06-2X/def2-SVP.

\begin{tabular}{ccc}
\hline Bond No. & Bond Distance $(\AA)$ & Wiberg Bond Index \\
\hline $1(B-N)$ & 1.488 & 0.807 \\
$2(\mathrm{C}-\mathrm{B})$ & 1.547 & 1.037 \\
$3(\mathrm{C}-\mathrm{P})$ & 1.721 & 1.062 \\
$4(\mathrm{~N}-\mathrm{P})$ & 1.696 & 0.781 \\
$5(\mathrm{~B}-\mathrm{B})$ & 1.715 & 0.962
\end{tabular}




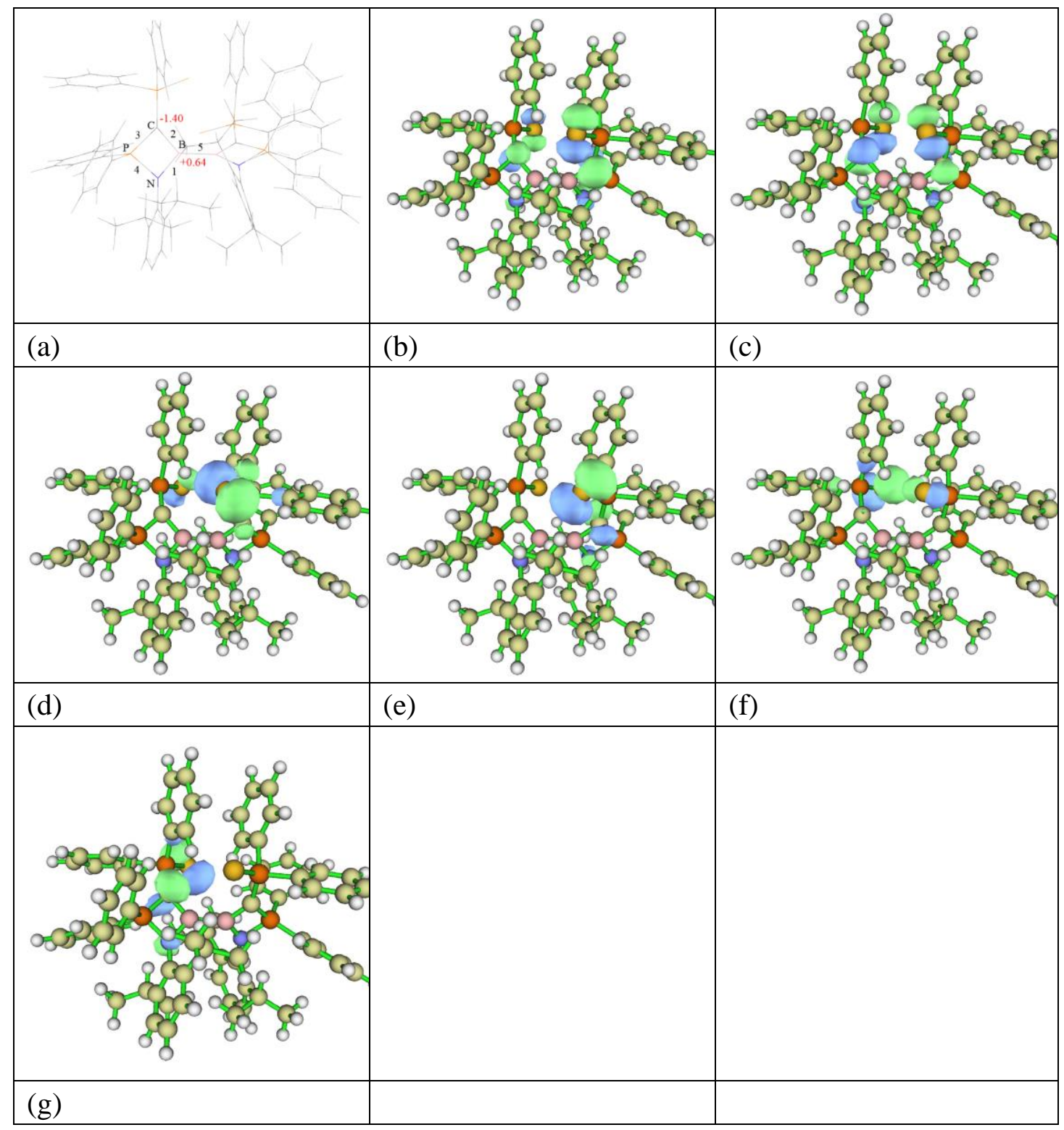

Figure S29. (a) Natural charge population of compound 5. Natural charges on C, B were shown in red. (b) HOMO (-6.46 eV). (c) HOMO-1 (-6.59 eV). (d) HOMO-2 ($6.75 \mathrm{eV}$ ). (e) HOMO-3 (-6.86 eV). (f) HOMO-4 (-6.92 eV). (g) HOMO-5 (-7.13 eV). B: pink, P: orange, S: dark yellow, C: yellow, H: white. 
Table S7. NBO analysis of compound $\mathbf{5}$.

\begin{tabular}{|c|c|c|c|c|}
\hline & Orbital & Occupancy & Contribution & Atomic Orbitals \\
\hline $1(\mathrm{~B}-\mathrm{N}, \sigma)$ & & 1.95 & $\begin{array}{l}19.99 \% \mathrm{~B}+ \\
80.01 \% \mathrm{~N}\end{array}$ & $\begin{array}{l}\text { B: } 25.93 \% \mathrm{~s}+ \\
73.73 \% \mathrm{p} \\
\mathrm{N}: 39.40 \% \mathrm{~s}+ \\
60.56 \% \mathrm{p}\end{array}$ \\
\hline $2(\mathrm{C}-\mathrm{B}, \sigma)$ & & 1.92 & $\begin{array}{l}71.08 \% \mathrm{C}+ \\
28.92 \% \mathrm{~B}\end{array}$ & $\begin{array}{l}\mathrm{C}: 39.20 \% \mathrm{~s}+ \\
60.73 \% \mathrm{p} \\
\mathrm{B}: 31.76 \% \mathrm{~s}+ \\
68.09 \% \mathrm{p}\end{array}$ \\
\hline $2(\mathrm{C}-\mathrm{B}, \pi)$ & & 1.78 & $\begin{array}{l}88.03 \% \mathrm{C}+ \\
11.97 \% \mathrm{~B}\end{array}$ & $\begin{array}{l}\text { C: } 99.81 \% p \\
\text { B: } 99.48 \% p\end{array}$ \\
\hline $3(\mathrm{C}-\mathrm{P}, \sigma)$ & & 1.96 & $\begin{array}{l}59.32 \% \mathrm{C}+ \\
40.68 \% \mathrm{P}\end{array}$ & $\begin{array}{l}\mathrm{C}: 24.75 \% \mathrm{~s}+ \\
75.15 \% \mathrm{p} \\
\mathrm{P}: 31.10 \% \mathrm{~s}+ \\
68.37 \% \mathrm{p}\end{array}$ \\
\hline $4(\mathrm{~N}-\mathrm{P}, \sigma)$ & & 1.95 & $\begin{array}{l}72.51 \% \mathrm{~N}+ \\
27.49 \% \mathrm{P}\end{array}$ & $\begin{array}{l}\mathrm{N}: 21.00 \% \mathrm{~s}+ \\
78.93 \% \mathrm{p} \\
\mathrm{P}: 19.88 \% \mathrm{~s}+ \\
78.92 \% \mathrm{p}\end{array}$ \\
\hline $5(\mathrm{~B}-\mathrm{B}, \sigma)$ & & 1.97 & $\begin{array}{l}49.95 \% \mathrm{~B}+ \\
50.05 \% \mathrm{~B}\end{array}$ & $\begin{array}{l}\text { B: } 42.22 \% \mathrm{~s}+ \\
57.76 \% \mathrm{p} \\
\mathrm{B}: 42.27 \% \mathrm{~s}+ \\
37.71 \% \mathrm{p}\end{array}$ \\
\hline
\end{tabular}




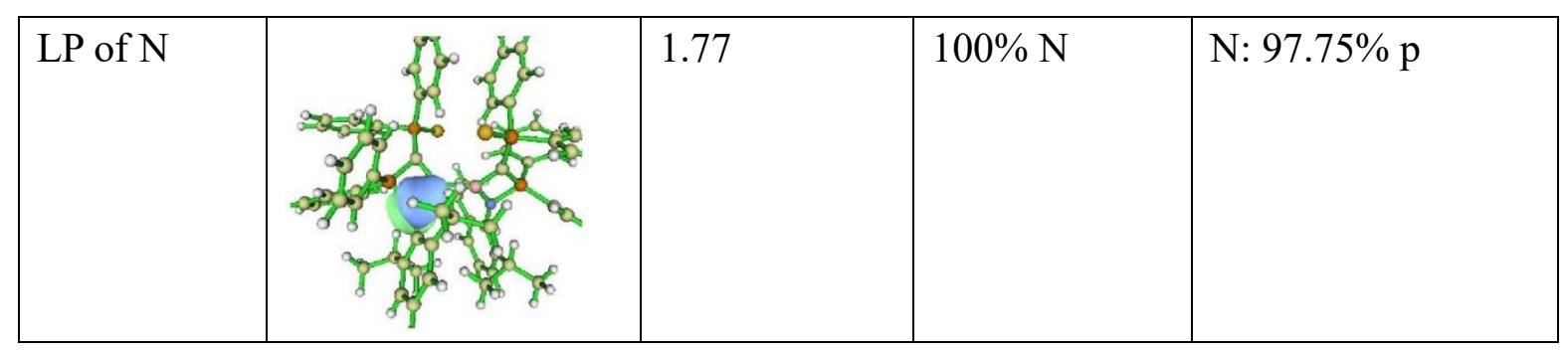




\section{S4. References}

1. Sheldrick, G. M. SADABS V2014/4 (Bruker AXS Inc.) University of Göttingen, Göttingen, Germany, 2014.

2. SHELXL-2014/6 (Sheldrick, 2014); Bruker AXS Inc., Madison, WI, USA, 2014.

3. Frisch, M. J. et al. Gaussian, Inc., Wallingford CT, 2016.

4. Lu, T.; Chen, F. J. Comput. Chem. 2012, 33, 580.

5. Glendening, E. D.; Badenhoop, J. K.; Reed, A. E.; Carpenter, J. E.; Bohmann, J. A.; Morales, C. M.; Landis, C. R.; Weinhold, F. NBO Version 6.0. 